\title{
Muon Fluence Measurements for Homeland Security Applications
}

Austin Ankney and Tim Berguson, Juniata College

James Borgardt, Juniata College Faculty

Richard T. Kouzes, PNNL Mentor

July 2010

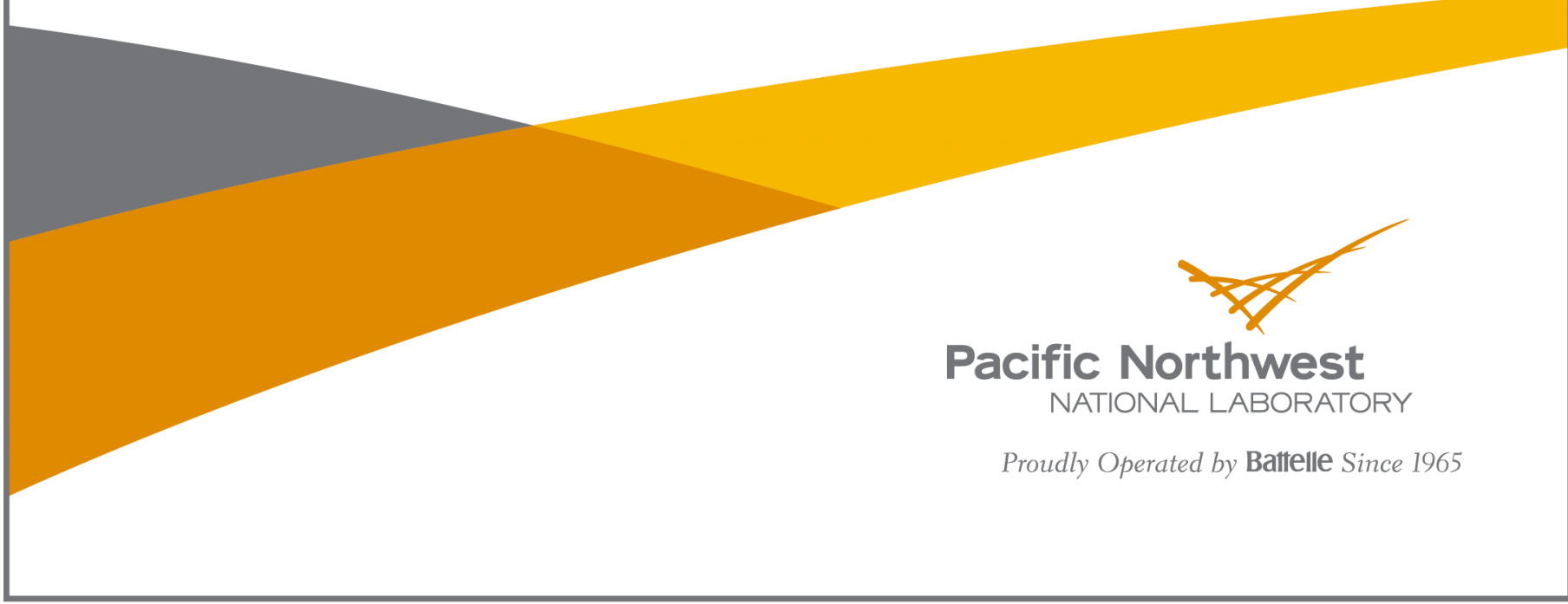




\section{DISCLAIMER}

This report was prepared as an account of work sponsored by an agency of the United States Government. Neither the United States Government nor any agency thereof, nor Battelle Memorial Institute, nor any of their employees, makes any warranty, express or implied, or assumes any legal liability or responsibility for the accuracy, completeness, or usefulness of any information, apparatus, product, or process disclosed, or represents that its use would not infringe privately owned rights. Reference herein to any specific commercial product, process, or service by trade name, trademark, manufacturer, or otherwise does not necessarily constitute or imply its endorsement, recommendation, or favoring by the United States Government or any agency thereof, or Battelle Memorial Institute. The views and opinions of authors expressed herein do not necessarily state or reflect those of the United States Government or any agency thereof.

PACIFIC NORTHWEST NATIONAL LABORATORY

operated by

BATTELLE

for the

UNITED STATES DEPARTMENT OF ENERGY

under Contract DE-AC05-76RL01830

Printed in the United States of America

Available to DOE and DOE contractors from the

Office of Scientific and Technical Information,

P.O. Box 62, Oak Ridge, TN 37831-0062;

ph: (865) 576-8401

fax: (865) 576-5728

email: reports@adonis.osti.gov

Available to the public from the National Technical Information Service, U.S. Department of Commerce, 5285 Port Royal Rd., Springfield, VA 22161

ph: (800) 553-6847

fax: (703) 605-6900

email: orders@ntis.fedworld.gov

online ordering: http://www.ntis.gov/ordering.htm

\section{ACKNOWLEDGEMENT}

This work was supported by the US Department of Energy, National

Science Foundation, and Pacific Northwest National Laboratory 
PNNL- 19632

\title{
Muon Fluence Measurements for Homeland Security Applications
}

\author{
AS Ankney \\ TJ Berguson \\ JD Borgardt \\ RT Kouzes
}

July 22, 2010

Pacific Northwest National Laboratory

Richland, Washington 99352 


\section{Executive Summary}

Over the past decade radiation portal monitors (RPM), which have the capability to passively detect gamma ray and neutron radiation, have been deployed at border crossings, airports, and sea ports, diminishing the possibility of illicit nuclear materials entering the country. One challenge that occurs with operational RPMs is false-positive readings due to fluctuations in background radiation and environmental conditions. An enhanced understanding of the origin of different contributors to the RPM backgrounds could lead to improved detector design, alarm algorithms, and system efficacy.

This report focuses on work conducted at Pacific Northwest National Laboratory to better characterize aspects of backgrounds in RPMs deployed for homeland security purposes. Two polyvinyl toluene scintillators were utilized with supporting NIM electronics to measure the muon coincidence rate. Muon spallation is one mechanism by which background neutrons are produced. The measurements performed concentrated on a broad investigation of the dependence of the muon flux on a) variations in solid angle subtended by the detector; b) the detector inclination with the horizontal; c) depth underground; and d) diurnal effects. These tests were conducted inside at Building 318/133, outdoors at Building 331G, and underground at Building 3425 at Pacific Northwest National Laboratory.

Results of the analysis elucidated muon behavior for all tests. The muon flux in above ground locations decreased linearly as the detector separation increased, thus correlating with the solid angle subtended. Muon fluence followed a $\cos ^{2.3} \theta$ dependence when inclination varied, where $\theta$ is defined with respect to the vertical. This finding is greater than values of $1.85 \pm 0.10$ cited by referenced studies, indicating our data exhibited a more pronounced reduction in the count rate with increasing angle. The muon attenuation underground, measured at one depth at the underground laboratory at Building 3425, was consistent with earlier measurements indicating a depth of 35-40 meters water equivalent. The diurnal behavior of the muon flux exhibited both periodic and aperiodic intervals, each on the order of days, for data collected over a seven-day period. 


\section{Acronyms and Abbreviations}

$\begin{array}{ll}\text { cps } & \text { Counts per second } \\ \text { CR } & \text { Cosmic radiation } \\ \text { FWHM } & \text { Full width at half max } \\ \text { GEANT } & \text { GEometry ANd Tracking (simulation software package) } \\ \text { MWE } & \text { Meters Water Equivalent } \\ \text { NIM } & \text { Nuclear Instrument Module } \\ \text { NORM } & \text { Naturally Occurring Radioactive Materials } \\ \text { PNNL } & \text { Pacific Northwest National Laboratory } \\ \text { PMT } & \text { photomultiplier tube } \\ \text { PNNL } & \text { Pacific Northwest National Laboratory } \\ \text { PVT } & \text { polyvinyl toluene (a.k.a., "plastic") scintillator } \\ \text { RPM } & \text { radiation portal monitor }\end{array}$




\section{Contents}

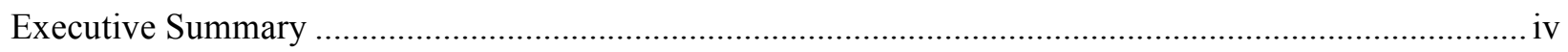

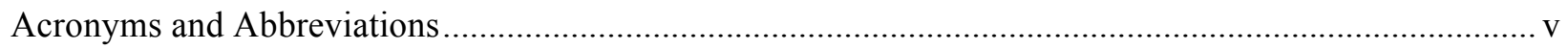

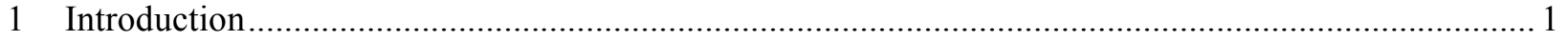

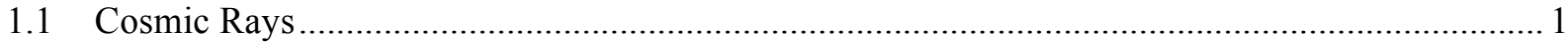

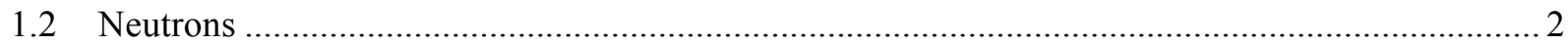

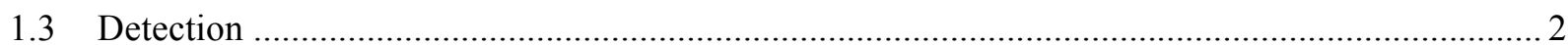

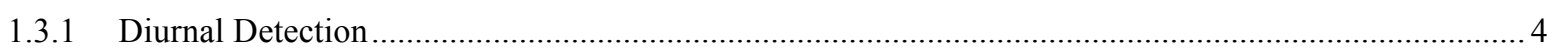

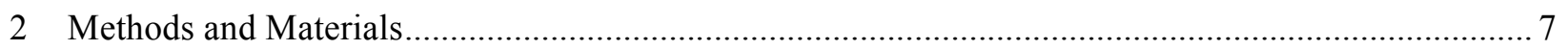

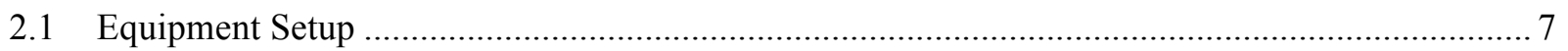

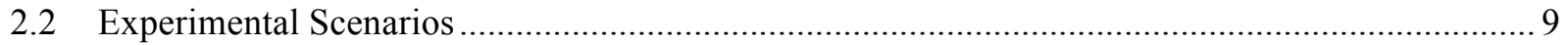

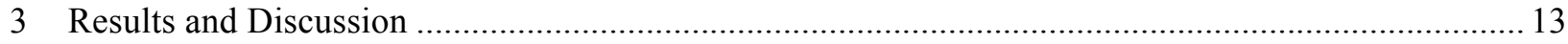

3.1 Effects of Scintillation Separation Testing on Muon Rates .................................................... 13

3.2 Effects of Scintillation Orientation Testing on Muon Rates ...................................................... 14

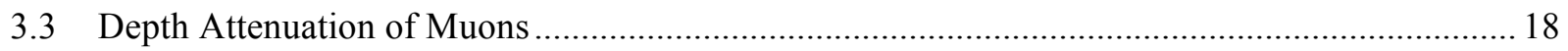

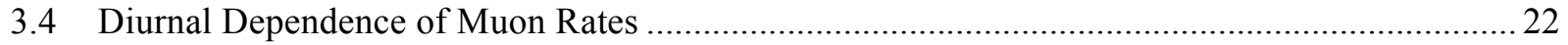

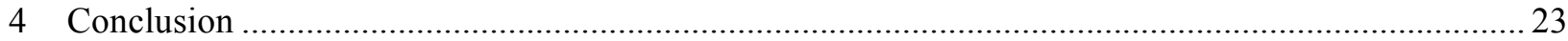

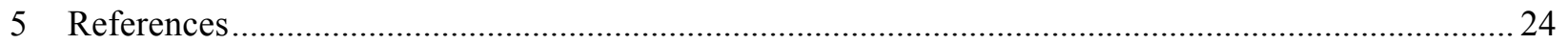

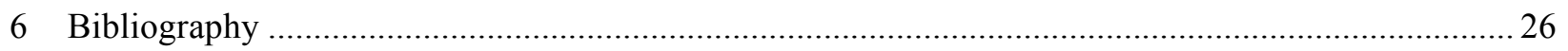




\section{Figures and Tables}

\section{Figures}

Figure 1 - Simplified illustration of cosmic ray interaction within the atmosphere. Actual showers generate on the order of $10^{9}$ particles and are narrowly confined along the path of the incident cosmic ray.......

Figure 2 - Relevance of different photon interaction mechanisms in PVT. Photo-absorption dominates below $20 \mathrm{keV}$; Compton scattering between $20 \mathrm{keV}$ and $20 \mathrm{MeV}$; and pair production at energies above $20 \mathrm{MeV}$ [Ely et al. (2004)]. Note the plot depicts only energies up to $10 \mathrm{keV}-10 \mathrm{MeV}$ as this is the energy regime generally of interest for illicit nuclear materials.

Figure 3 - Gamma ray and muon peaks overlap in a spectrum taken from a PVT scintillator. The solid lines represent approximate contributions from each component to the composite spectrum.

Figure 4 - Example of diurnal variation of gamma and neutron radiation, collected at latitude $46.285 \mathrm{~N}$ [Kouzes et al. (2008b)]

Figure 5 - Variation in cosmic rays at different latitudes [Thompson (1938)]. Variation from the mean is less than 5\% at all latitudes shown. Data are represented by dots; solid lines depict computational fits.

Figure 6 - Representative alignment of parallel PVT panels. Insulation sheathing was used to vary the panel separation and provide stability.

Figure 7 - Supporting electronics using NIM modules for the two PVT panels in coincidence................ 8

Figure 8 - The full singles spectrum, including gamma ray and muon contributions, before coincidence was set (unshaded), and the coincident muon spectrum (shaded) after coincidence was required to reduce the gamma ray contribution.

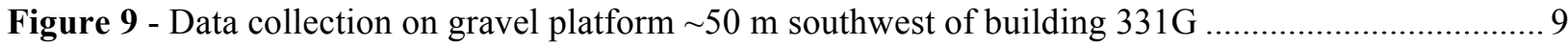

Figure 10 - Schematic of Deep Lab at building 3425. Data was collected in the rest room space.......... 10

Figure 11 - In order to vary the angle of the detector, insulation was placed beneath one side of the bottom detector to increase the angle.

Figure 12 - Geometry used to determine solid angle. Point $\mathrm{P}$ lies at the geometric center of the two panels with the origin lying at the center of the upper surface of the lower panel.

Figure 13 - Displays data and solid angle over a range of PVT panel separations. The error bars are too small to show up clearly on the plots.

Figure 14 - As solid angle decreases with increasing panel separation, the muon count rate decreases. Accidental coincidences were subtracted from the muon count rate. The dashed line is an extrapolated linear fit giving a y-intercept of $3.7 \mathrm{cps}$.

Figure 15 - Data collected at the two above ground locations, with orientations in both north-south and east-west alignments. The error bars are approximately the same size as the data point markers.... 15

Figure 16 - Above ground data with the horizon angle corrected. The error bars were approximately the same size as the data point markers. 
Figure 17 - Data from present study taken in an east-west alignment compared to scaled results from Lin, et al. The error bars were approximately the same size as the data point markers. 16

Figure 18 - North-south and east-west data with orientation testing completed underground. 16

Figure 20 - Composite display of all uncorrected, with no horizon cutoff adjustment, data runs for varying inclinations above ground and underground. The error bars were approximately the same size as the data point markers.

Figure 21 - Comparison of experimental data to that of cosine curves were placed together to compare our indoor data to Grieder's data.

Figure 22 - Surface earthen berm from southeast of Building 3425 in August 2009 during construction.

Figure 23 - Surface berm of Building 3425 in July 2010 at completion of construction. Note additional rock cap earthen bunker compared to Figure 22.

Figure 24 -Data from several studies of muon attenuation at shallow depth. The fit from a numerical model developed by Barbouti et al. for shallow depths is given by the dotted line [Barbouti et al. (1983)].

Figure 25 - Data presented by Effremenko, along with data collected at the underground lab in 3425 during August 2009 and July 2010. The solid line is the best fit for data generated by a GEANT simulation performed by Effremenko. August 2009 error bars are larger due to poorer statistics relative to the July 2010 data.

Figure 26 - The muon count rate over an eight day period. The shaded red and blue regions denote 24hour intervals. The approximate times for daily minima and maxima in the periodic phase are indicated.

Tables

Table 1: Summary of data comparing muon fluence at surface to that in the 3425 Deep Lab 


\section{Introduction}

Over the past decade, coordinated work has been initiated in an effort to thwart any attempts to bring illicit nuclear material into the United States. The US has employed a layered defense posture, which, among other initiatives, includes efforts to secure nuclear material and install passive radiation detectors at international and domestic ports of entry. The majority of such deployed detectors are radiation portal monitor (RPM) systems utilizing polyvinyl toluene (PVT) based detectors, which have the capacity to sense gamma rays, and pressurized ${ }^{3} \mathrm{He}$ tubes sheathed in a moderator to register neutrons. Natural background gamma ray count rates observed in such systems are on the order of thousands of counts per second, and algorithms are used to generate an alarm if gamma ray count rates rise above a preset threshold.

One of many challenges in this scheme is that, while illegal nuclear materials of interest emit gamma radiation, there are numerous benign naturally occurring radioactive materials (NORM) carried in common commercial products that do so as well, including ceramics, fertilizers, and kitty litter, among others. However, such NORM does not emit neutrons while the threat material plutonium does, and thus neutron counts above natural background are highly suggestive of a material warranting further scrutiny. Since the natural background rate from cosmic ray neutrons is only a few per second, statistics are much poorer than for gamma rays, and it is critical to measure and categorize the background neutron fluence as accurately as possible.

\subsection{Cosmic Rays}

Cosmic rays originate from extraterrestrial sources and are composed primarily of protons $(90 \%)$, with lesser contributions from alpha particles $(9 \%)$, electrons (1\%) and trace amounts of heavier nuclei and gamma rays [Grieder (2001)]. Primary cosmic rays are emitted by extragalactic sources such as supernovae and can interact with interstellar matter generating secondary cosmic rays. Within our solar system, the sun can also generate low energy cosmic rays during solar flares. Upon entering the earth's atmosphere, cosmic rays can interact with atmospheric molecules like oxygen and nitrogen creating air showers of subatomic particles including pions, which can quickly decay in secondary showers generating muons, neutrons and other particles. A simplified illustration is given in Figure 1; an actual shower event can generate on the order of $10^{9}$ particles, which are narrowly confined along the path of the incident cosmic ray. The amount of primary cosmic rays and their secondary progeny at the earth's surface is a function of latitude, elevation, and the surrounding environment [Kouzes et al. (2008a)].

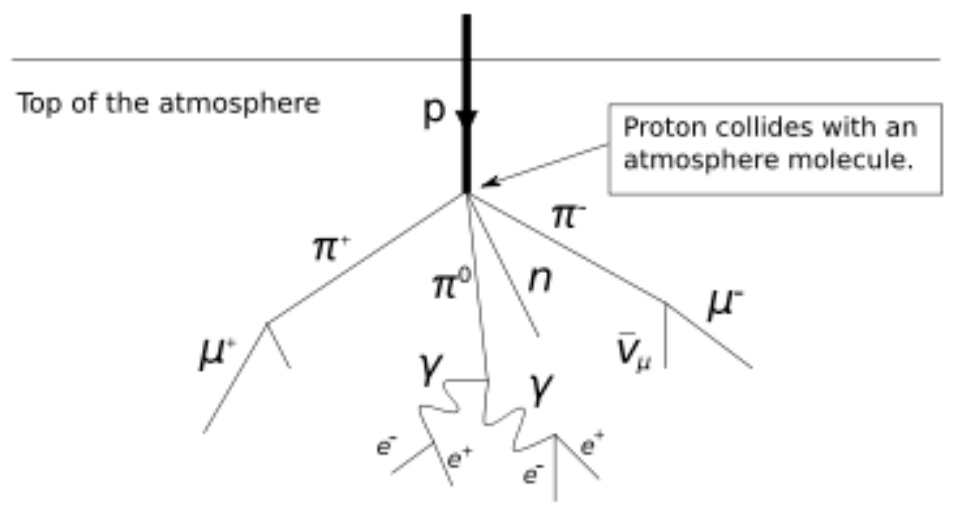

Figure 1 - Simplified illustration of cosmic ray interaction within the atmosphere. Actual showers generate on the order of $10^{9}$ particles and are narrowly confined along the path of the incident cosmic ray. 
Muons, generated in the atmosphere in secondary showers, have a mean lifetime of about $2.2 \mu \mathrm{s}$, sufficient to reach the earth's surface, leading to a muon flux at sea level of approximately 170 counts $/\left(\mathrm{m}^{2} \cdot \mathrm{s}\right)$ in the United States [Grieder (2001)].

\subsection{Neutrons}

The origin and characteristics of the neutron background at earth is not well understood. There are several known contributors to the neutron signature at the earth's surface. The primary source arises from cosmic ray-induced secondary particles including a small contribution from muon-induced spallation and primarily neutron-induced spallation, the so-called "ship effect" [Kouzes et al. (2008a)]. Other less prominent sources include nuclear decay and solar activity. This work is motivated by a paucity of work done separating the origins of neutrons at the surface of the earth. This lack of knowledge, coupled with a low neutron background rate, render neutron-based detection efforts susceptible to fluctuations in the neutron background. An improved understanding of the relative contributions of muon spallation and neutron spallation to the detected neutron counts could help optimize detector and instrumentation design, alarm algorithms and computer modeling, thus augmenting detection efforts.

\subsection{Detection}

Plastic scintillators such as PVT-based detectors utilize scintillation as the response to ionizing radiation interactions. Such detection systems are made of two optically coupled components: a liquid or solid scintillating material which fluoresces to produce light when incident radiation deposits energy; and a photomultiplier which converts and amplifies this low intensity light pulse, using a series of dynode stages, into a measureable electrical current, thereby allowing individual photons to be detected. This signal is fed into electronics that can discriminate between different types of radiation and between different energies of the same radiation. [Knoll (2000)]

Three photon interaction mechanisms can occur in PVT: Photo-absorption, Compton scattering, and pair production. In the photoelectric absorption process the photon energy is completely transferred to the absorber atom and an energetic photoelectron is emitted from an atomic bound shell. The energy of the ejected electron is that of the incident photon, less the binding energy of the photoelectron. Thus for incident photon energies above several hundred $\mathrm{keV}$ the photoelectron acquires the bulk of the photon energy. In a Compton scattering interaction, an incident gamma ray photon is deflected through an angle $\theta$ from its incident path by an electron, assumed to be at rest, in the detecting material. The photon transfers a fraction of its energy, depending on this scattering angle, to this recoil electron. The energy transfer is least for small scattering angles, $\theta \approx 0$, and increases for larger scattering angles as $\theta$ approaches $\pi$. Pair production occurs when the energy of the incident gamma ray exceeds twice the rest mass energy of an electron, resulting in the creation of an electron-positron pair whose kinetic energy equals the excess energy above the $1.02 \mathrm{MeV}$ required to create the pair. Due to this energy threshold pair production is prevalent for interactions with high-energy photons.

Each of these three processes is dominant over a specific energy range. Photo-absorption dominates below $\sim 20 \mathrm{keV}$; Compton scattering between $\sim 20 \mathrm{keV}$ and $\sim 20 \mathrm{MeV}$; and pair production at energies above $\sim 20 \mathrm{MeV}$. The photon interaction probabilities in PVT are given in Figure 2 [Ely et al. (2004)] for energies between $10 \mathrm{keV}$ and $10 \mathrm{MeV}$. These limits were chosen as they represent the typical energy regime of radioisotope sources. Compton scattering is the dominant interaction mechanism in 
PVT detectors between $\sim 20 \mathrm{keV}$ to $\sim 20 \mathrm{MeV}$, the energy range generally of interest for illicit nuclear materials.

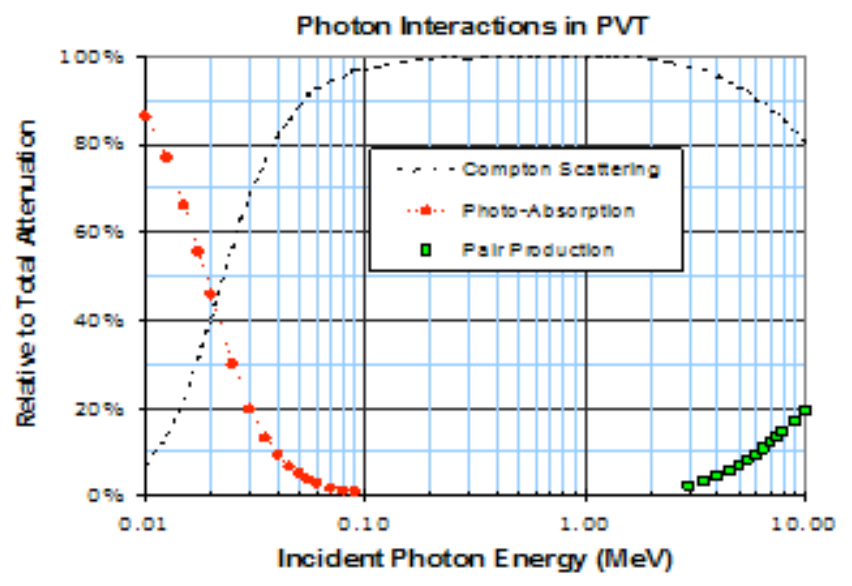

Figure 2 - Relevance of different photon interaction mechanisms in PVT. Photo-absorption dominates below $20 \mathrm{keV}$; Compton scattering between $20 \mathrm{keV}$ and $20 \mathrm{MeV}$; and pair production at energies above $20 \mathrm{MeV}$ [Ely et al. (2004)]. Note the plot depicts only energies up to $10 \mathrm{keV}-10 \mathrm{MeV}$ as this is the energy regime generally of interest for illicit nuclear materials.

Compton scattered gamma rays therefore generally deposit a variable portion of their full energy before exiting the scintillator, leading to low resolution. Muons however, traveling at velocities approaching the speed of light, act as minimum ionizing particles depositing a fixed amount of energy, $\sim 2$ $\mathrm{MeV} / \mathrm{cm}$ [Knoll (2000)], when fluorescing photons within the detecting medium leading to a broad peak at about $10 \mathrm{MeV}$ for a $5 \mathrm{~cm}$ thick plastic scintillator with a $\sim 1 \mathrm{~g} / \mathrm{cm}^{2}$ areal density. This causes a small overlap in the PVT acquired spectra of high-energy gamma rays and the much higher energy muons (Figure 3). No formal energy calibration was performed. 
PNNL- 19632

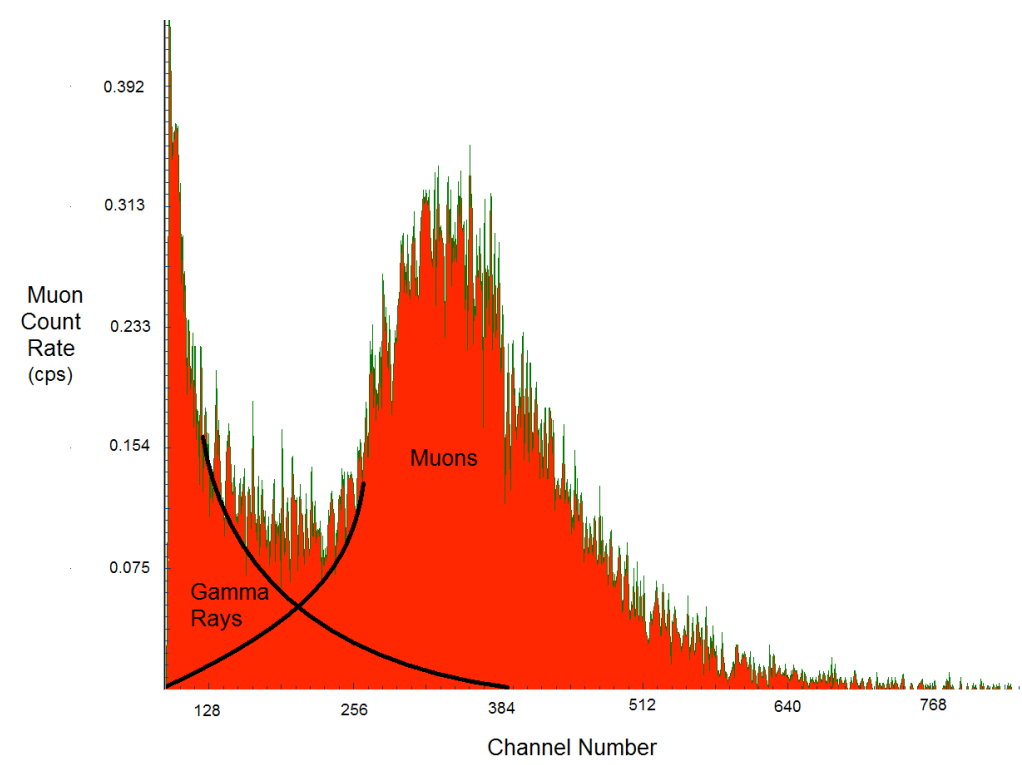

Figure 3 - Gamma ray and muon peaks overlap in a spectrum taken from a PVT scintillator. The solid lines represent approximate contributions from each component to the composite spectrum.

\subsubsection{Diurnal Detection}

Solar diurnal variations have been reported in gamma, neutron and muon fluxes [Mailyan et al. (2010); Tiwari et al. (2008)]. Variations in neutron flux are greater at higher latitudes relative to lower latitudes. The amplitudes of the neutron daily variations, corresponding to the lowest energy primary protons, are comparable with the magnitudes of variations of charged secondary cosmic rays [Mailyan et al. (2010)]. The diurnal variations of gamma rays, both low and high energy, and neutron radiation at the earth's surface at latitude $46.285 \mathrm{~N}$ are shown below (Figure 4) [Kouzes et al. (2008b)]. Both the total gamma ray and total neutron oscillations are noted to peak in the late evening hours, and reach a minimum in the late morning hours. The variation from the mean amounts to about $1 \%$ at the latitude of PNNL. 
PNNL- 19632
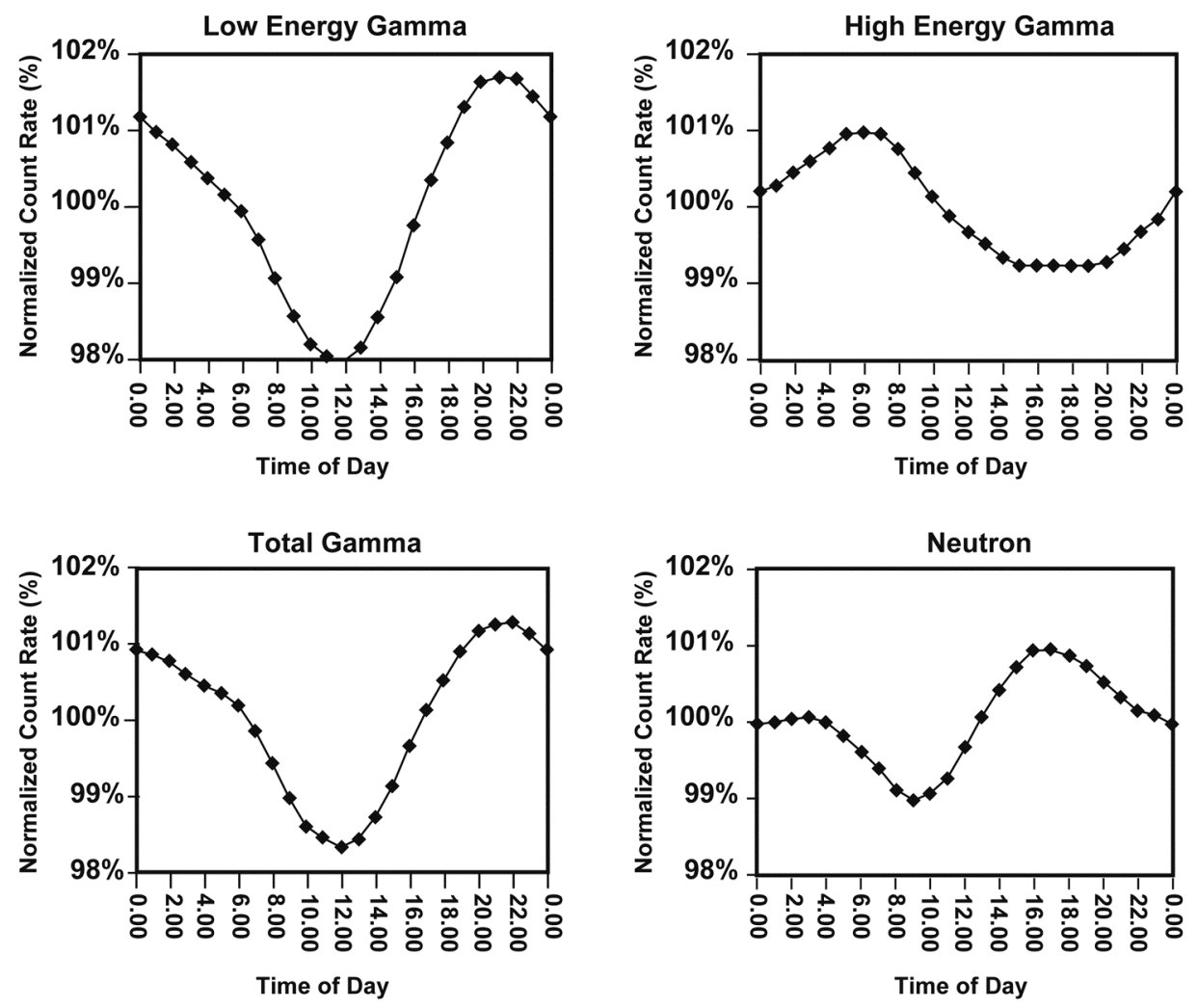

Figure 4 - Example of diurnal variation of gamma and neutron radiation, collected at latitude $46.285 \mathrm{~N}$ [Kouzes et al. (2008b)]

Muons have also been reported to display diurnal variations with low energy muon variations exhibiting more pronounced oscillations compared to higher energy muons. The amplitude of daily variations measured with ground-based detectors has been reported to be less than $0.5 \%$ at latitudes between $54.7 \mathrm{~N}$ and 40S (Figure 5) [Thompson (1938)]. 
PNNL- 19632

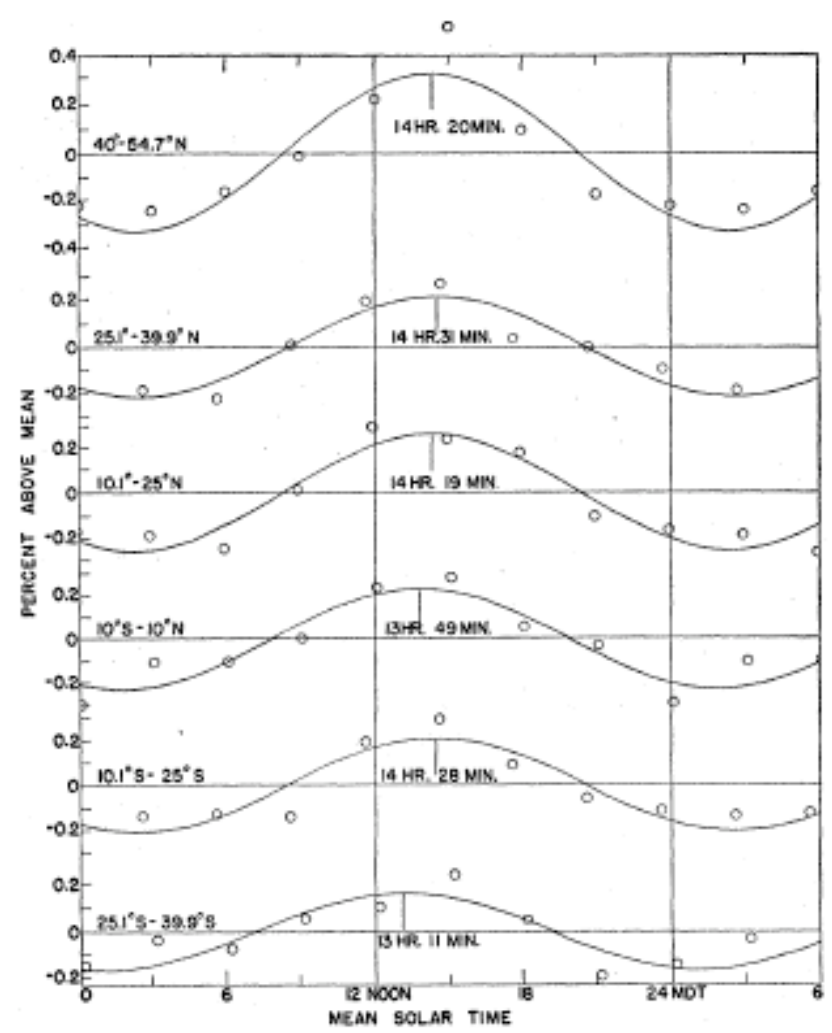

Figure 5 - Variation in cosmic rays at different latitudes [Thompson (1938)]. Variation from the mean is less than $5 \%$ at all latitudes shown. Data are represented by dots; solid lines depict computational fits.

While extragalactic cosmic radiation impinges isotropically on the earth, the day-night variation may be due to the changing morphology of the earth's magnetic field and its shaping by the solar wind. However, the effect depends heavily on the energy of the primary. Gamma and neutron radiation is produced by relatively low energy primary cosmic rays in the 1-20 GeV range, an energy not likely to produce muons. Muons detected at sea level have an energy distribution that peaks at around $3 \mathrm{GeV}$, suggesting an energy of $5 \mathrm{GeV}$ at the top of the atmosphere, meaning the energies of the primaries, which create the pions that decay to muons, are much higher than the 1-20 GeV range. The neutrons come from much lower energy primaries than the muons, and the diurnal effects and Forbush decreases of relatively local fluctuations in earth's magnetic field on low energy primaries should therefore be much stronger than for muons. Since primary cosmic rays leading to muon generation are of much higher energy than those causing gamma rays and neutrons, their observed diurnal variation, $\sim 1 \%$ from the mean, is somewhat suppressed compared to that of muons, $<0.5 \%$ from the mean. 


\section{Methods and Materials}

Two large PVT panels $(1.20 \mathrm{~m} \times 0.4 \mathrm{~m} \times 0.05 \mathrm{~m})$ were interfaced with supporting electronics to measure coincident muon counts. Data were collected under a number of conditions varying panel separation, inclination, orientation, and location. The equipment is discussed in this section and the results from these investigations are discussed in section 3 .

\subsection{Equipment Setup}

Two stacked PVT scintillators were utilized with their large faces oriented parallel (see Figure 6 for representative alignment). Slabs of commercial extruded polystyrene insulated sheathing, $10.2 \mathrm{~cm}$ thick, were used to stabilize the panels and vary their separation. A photomultiplier tube was affixed to the end of each panel as shown.

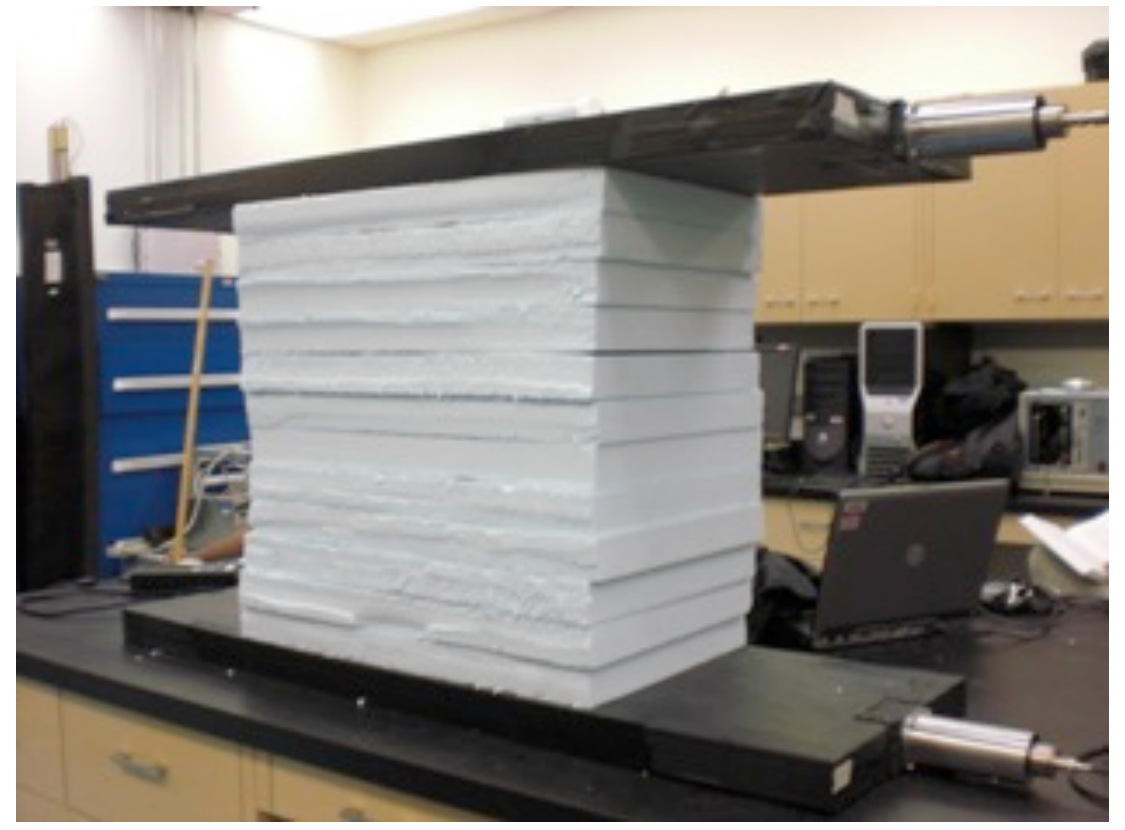

Figure 6 - Representative alignment of parallel PVT panels. Insulation sheathing was used to vary the panel separation and provide stability.

The same electrical equipment was used for all experimental work unless otherwise noted. The PMT signal from each panel was directed to an Ortec 142AH preamplifier and supporting NIM electronics (Figure 7), which allowed singles and coincident counts to be registered. 


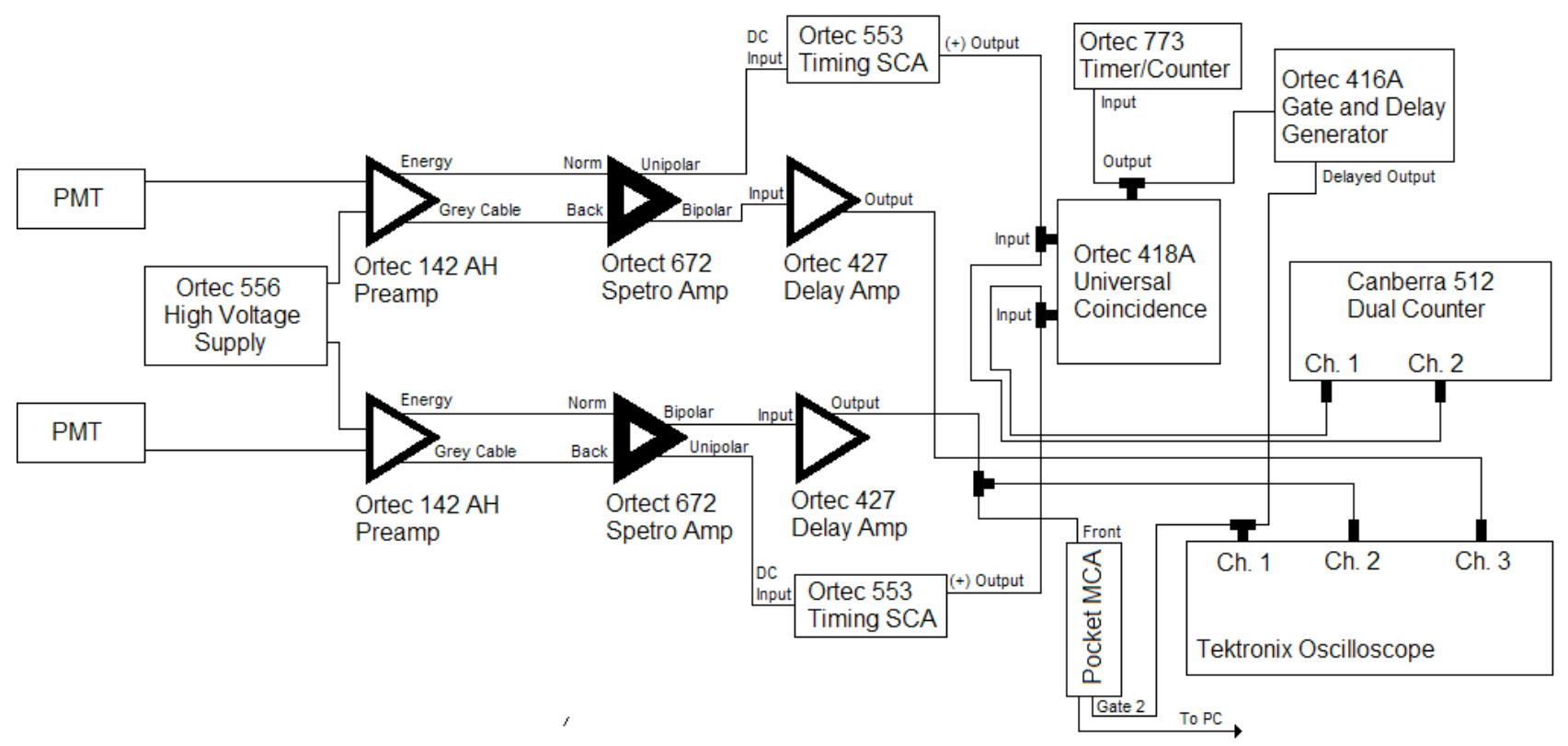

Figure 7 - Supporting electronics using NIM modules for the two PVT panels in coincidence.

In order to reduce the gamma ray contribution to the muon signal, the lower level discriminator was adjusted to channel 90, approximately the channel where the two appeared to be equal. The muon peak, estimated to be $\sim 10 \mathrm{MeV}$, lies near channel 300, so channel 90 correlates to a low level cutoff at about $3 \mathrm{MeV}$. Since there is an overlap in the two spectral contributions, this technique both omits some muons of interest, and includes some unwanted higher energy gamma rays. The discriminator was set in an attempt to offset these two opposing effects. The resulting spectrum is shown in Figure 8.

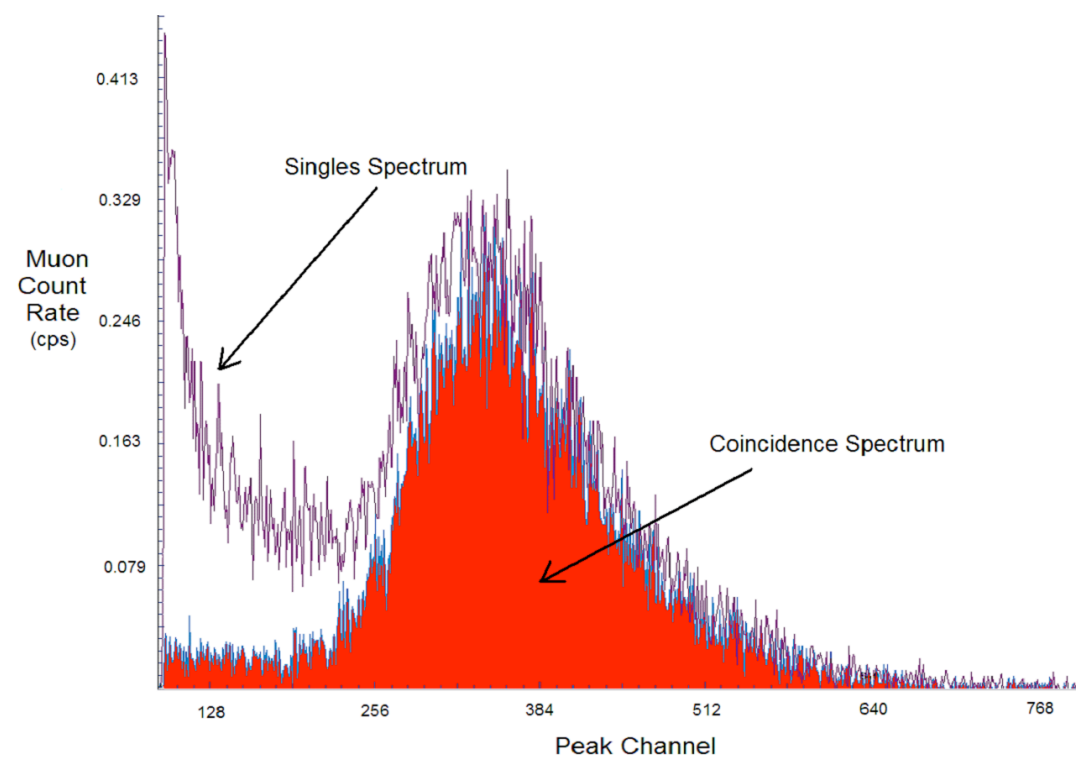

Figure 8 - The full singles spectrum, including gamma ray and muon contributions, before coincidence was set (unshaded), and the coincident muon spectrum (shaded) after coincidence was required to reduce the gamma ray contribution. 
Measurements were conducted at a single inclination of $0^{\circ}$ with the slab insulation used to vary the panel separation. Measurements were also conducted at varying inclinations and fixed panel separation. Data for these scenarios were collected in orientations approximating both east-west and north-south alignments relative to the axial symmetry of the PMTs. Measurements were carried out in several locations under varying environmental conditions and degrees of shielding including outside of building 331G, indoors at 318/133, and at building 3425 in the Deep Lab. Black fabric draped over the apparatus was employed as a light shield in all cases.

\subsection{Experimental Scenarios}

A series of muon coincidence measurements was conducted at the three sites (indoors, outdoors, subterranean) noted above, in east-west and north-south orientations. Environmental information is conveyed in Figure 6 (indoors at 331/133), Figure 9 (outside of 331G) and Figure 10 (schematic of 3425Deep Lab).

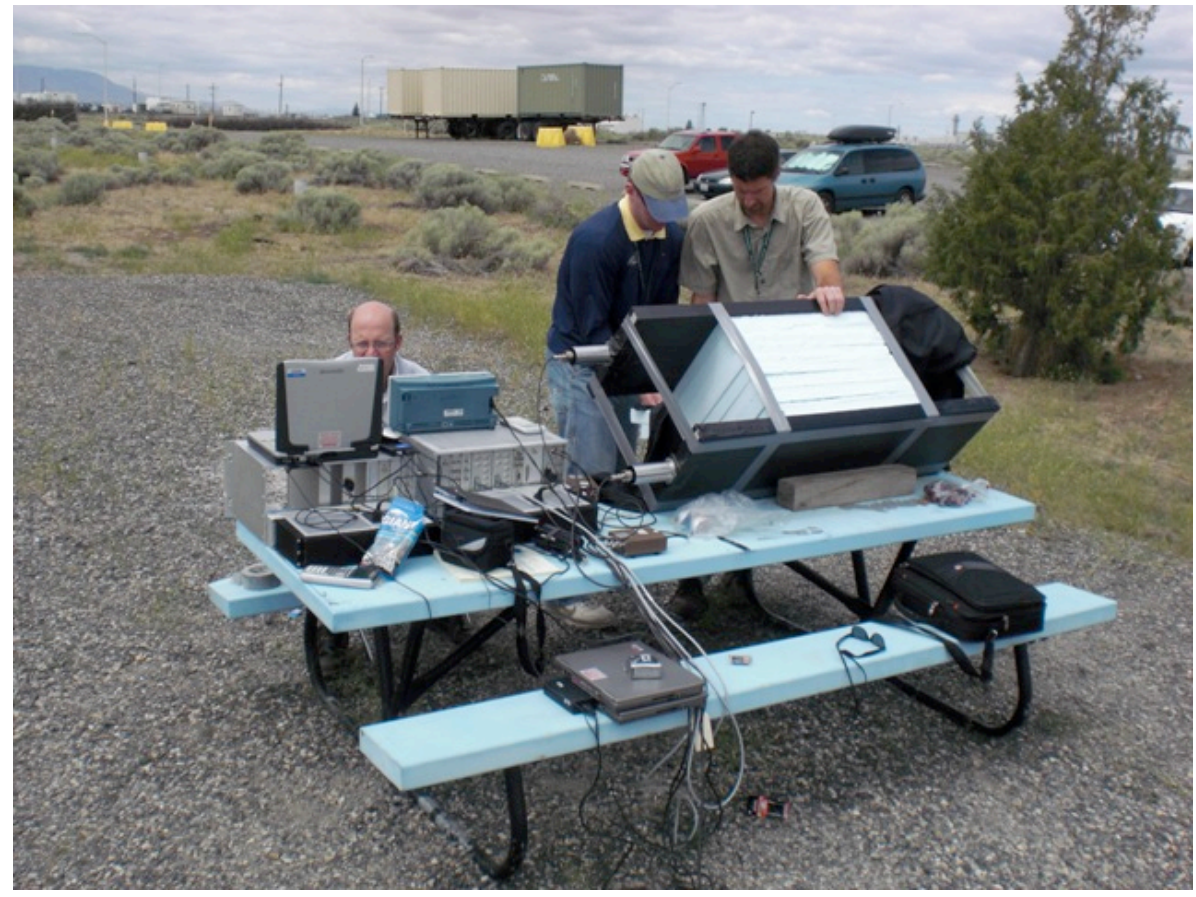

Figure 9 - Data collection on gravel platform $\sim 50$ m southwest of building $331 \mathrm{G}$ 
PNNL- 19632

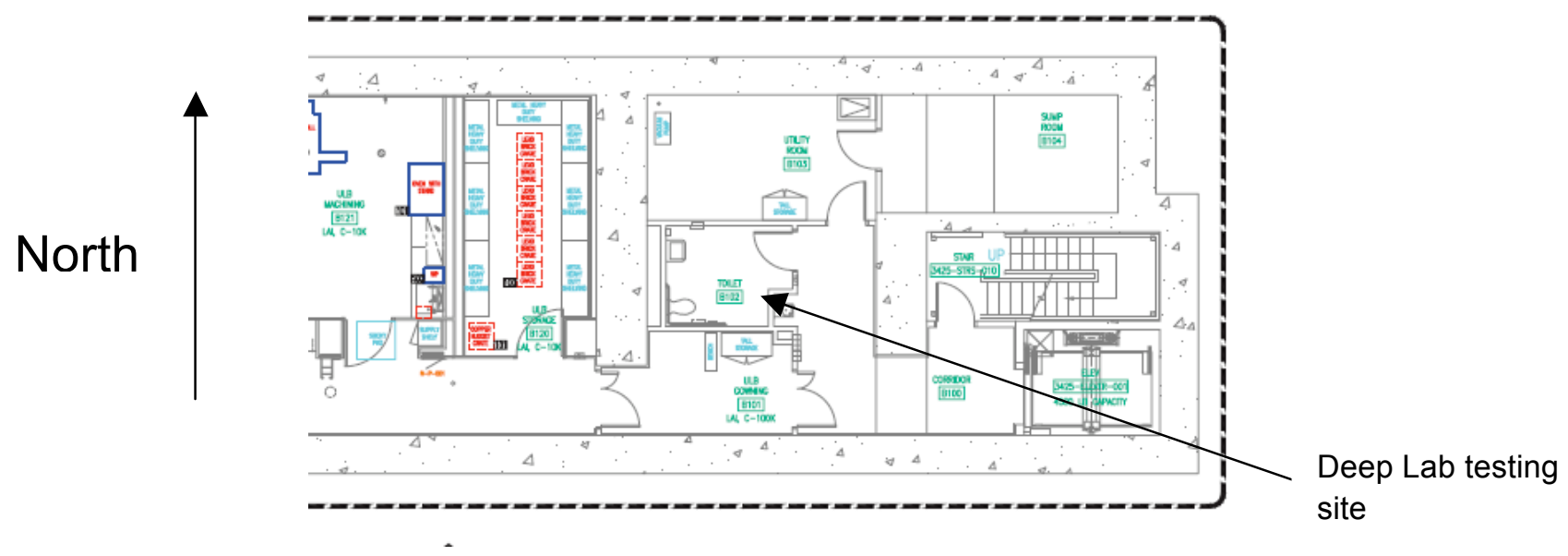

Figure 10 - Schematic of Deep Lab at building 3425. Data was collected in the rest room space.

\subsubsection{Effects of Scintillation Separation Testing on Muon Rates}

A series of experiments was conducted with the PVT panel faces aligned parallel to each other and in a horizontal plane (as shown in Figure 6). Slabs of $7-10 \mathrm{~cm}$ thick polystyrene sheathing were used to separate the panels by distances ranging between $0 \mathrm{~cm}$ and $61.4 \mathrm{~cm}$. This changed the effective Cartesian solid angle subtended by the rectangular scintillator faces, altering the detected muon flux. Muon coincidence data was collected over 3-minute intervals.

\subsubsection{Effects of Scintillation Orientation Testing on Muon Rates}

A second series of experiments was conducted with the PVT panel faces aligned parallel, but with a fixed separation of $61.4 \mathrm{~cm}$ with polystyrene sheathing, and the entire apparatus rotated with respect to the horizontal about the PMT's cylindrical symmetry axis. The panels were secured with duct-tape; wooden slats, cinderblocks, and spare sheathing were used to provide stability (Figure 11). Muon coincidence data were collected over 3-minute intervals, with the angle being incremented by $10^{\circ}-20^{\circ}$ between runs to cover absolute angles between $0^{\circ}$ (panel faces horizontal as in Figure 6) and $90^{\circ}$ (panel faces vertical). A series of measurements was conducted at the three sites noted above, in east-west and north-south orientations. 
PNNL- 19632

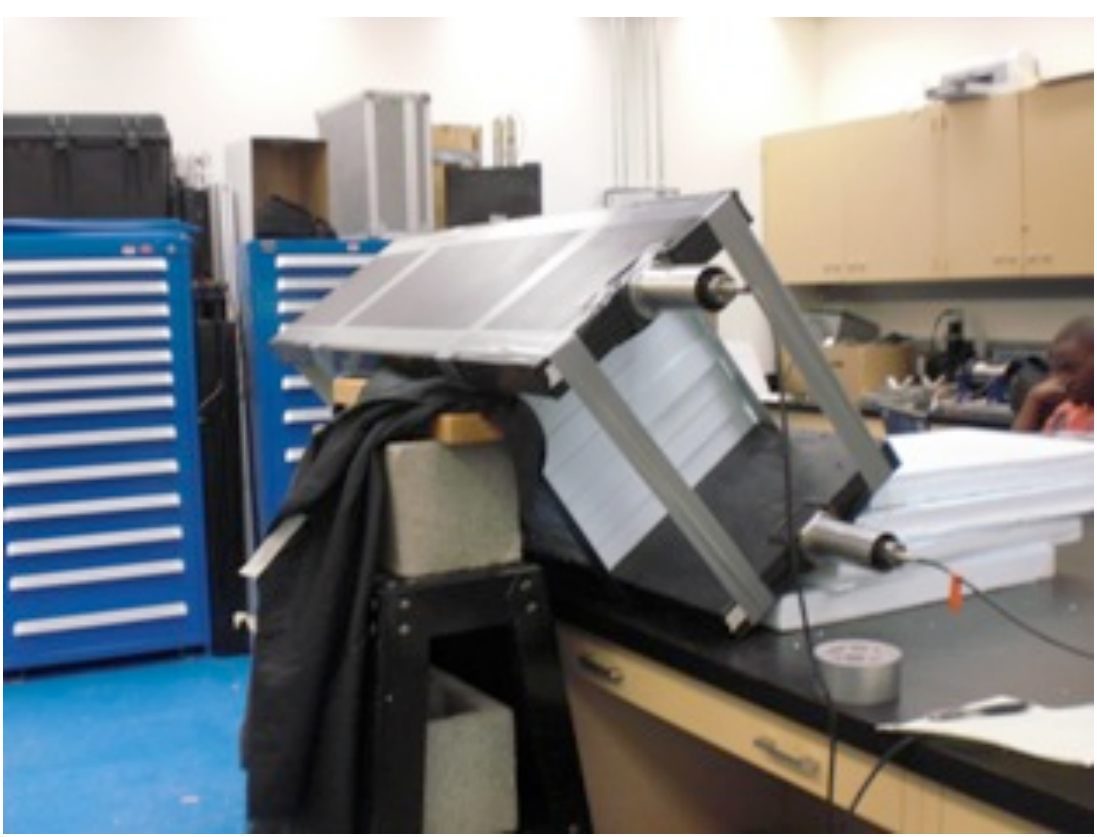

Figure 11 - In order to vary the angle of the detector, insulation was placed beneath one side of the bottom detector to increase the angle.

To determine the solid angle, the middle of the long detector face was taken to lie in the x-y plane with the perpendicular distance between the two panel faces being $2 z$. In a general case, the solid angle subtended by the rectangular scintillators from an arbitrary point above the detector is equal to the projection $\mathrm{dA}_{\mathrm{p}}$ of a differential area $\mathrm{dA}$ divided by the square of the distance between the solid angle and the area element (Figure 12):

$$
d \Omega=\frac{d A_{P}}{R^{2}}
$$

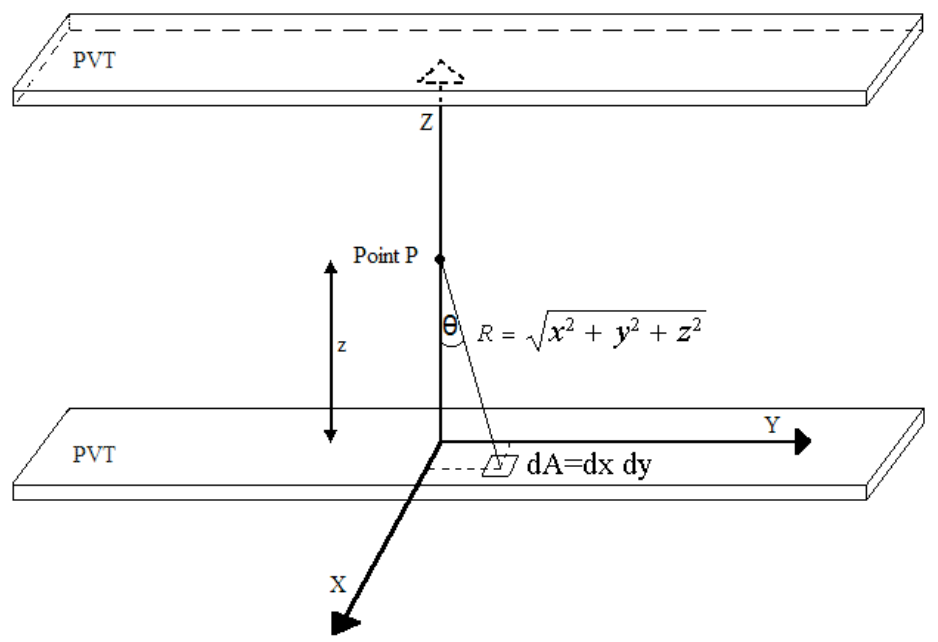

Figure 12 - Geometry used to determine solid angle. Point $\mathrm{P}$ lies at the geometric center of the two panels with the origin lying at the center of the upper surface of the lower panel. 
The differential of the projected area is equal to the cosine of the angle $\theta$ between the surface and the line of sight multiplied by the differential area,

$$
d A_{P}=\cos \theta \cdot d A=\frac{z}{R} \cdot d x d y
$$

where $\mathrm{z}$ represents the perpendicular distance above the plane midway between the two detector faces, and dxdy is the differential area dA. The differential solid angle is then given by

$$
d \Omega=\frac{d A_{P}}{R^{2}}=\frac{z}{R^{3}} d x d y=\frac{z}{\left(x^{2}+y^{2}+z^{2}\right)^{3 / 2}} d x d y
$$

Since the detector faces were aligned and the point $\mathrm{P}$ in Figure 12 is geometrically centered on midway between the panels, this expression can be more easily integrated taking advantage of symmetry. Calling the detector dimensions $\mathrm{W}$ and L, $40 \mathrm{~cm}$ and $120 \mathrm{~cm}$ respectively, yields [Pevey (1998)]

$$
\Omega=4 z \int_{0}^{L / 2} \int_{0}^{W / 2} \frac{1}{\left(x^{2}+y^{2}+z^{2}\right)^{3 / 2}} d x d y
$$

This expression was integrated using Mathematica 7.0 [Wolfram Research (2008)] was used to integrate all geometries tested.

The solid angle represents the acceptance angle that muons can enter within and pass through both scintillators, generating coincident detection. However, when increasing the inclination of the panels the solid angle eventually crosses with the horizon; this was found to occur at an inclination of $46.7^{\circ}$ with respect to the horizontal for the measurements in the underground facility where the panel separation was $40.8 \mathrm{~cm}$, and $57.9^{\circ}$ for measurements above ground where the separation was $61.4 \mathrm{~cm}$. Inclinations beyond these respective angles are obscured by the horizon, thus reducing the effective solid angle. Although theoretically a muon could still come from below the horizon, traveling through the ground, and pass through both scintillators, there are very few muons traveling upwards from this region due to a its significantly greater shielding. To account for this, the percent of the solid angle above the horizon was calculated, and the counts were multiplied by the inverse of the percentage in order to scale the counts to what they theoretically would have been if the entire angle were available. Note that this approach assumes equal muon flux originating below and above ground near the horizontal.

\subsubsection{Diurnal Dependence of Muon Rates}

The diurnal variation of cosmic radiation at earth was investigated by separating the PVT panels by a fixed $61.4 \mathrm{~cm}$ using the polystyrene sheathing as above. Muon coincidence data was taken over periods of at least 24-hours, with data extracted in 1-hour parcels. In this way any diurnal effects can be examined by comparing results of these 1-hour data sets over time. Data were collected indoors at $318 / 133$ with the detector in a north-south alignment. 


\section{Results and Discussion}

The results from the tests completed at 318/133 (indoors), 331G (outdoors), and 3425 (underground) are discussed in the following sections.

\subsection{Effects of Scintillation Separation Testing on Muon Rates}

Measurements varying the spacing of the parallel PVT scintillators were taken at two locations, inside at $318 / 133$ and outdoors about $50 \mathrm{~m}$ southwest of $331 \mathrm{G}$. The PVT scintillators were configured in a north-south orientation at 318 and an east-west orientation at $331 \mathrm{G}$. Eight to ten different panel spacing distances were tested at each location using the $\sim 10.2 \mathrm{~cm}$ (4") thick polystyrene slabs (Figure 6). As the spacing between the detectors is increased, the solid angle subtended by the detector decreased. This approximately, but not exactly, follows a $1 / \mathrm{R}^{2}$ dependence consistent with the rectangular detector geometry as discussed in section 2.2.2. Experimental results are presented in Figure 13 for various panel separations.

Initial indoor tests conducted with the two PVT panels lying flat and adjacent to each other and not overlapping, with their $120 \mathrm{~cm}$ sides parallel and separated by roughly $20 \mathrm{~cm}$, yielded count rates of roughly $1 \mathrm{cps}$ in each panel. This effectively represents an accidental count rate, as the two panels were not aligned while still in electronic coincidence, that was approximately $1-3 \%$ of the measured counts rate of typical experimental runs. Results, with accidental coincidence subtracted, are presented in Figure 13 for various panel separations. Error bars in the count rate are about $1 \%$, too small to be visible on the plot.

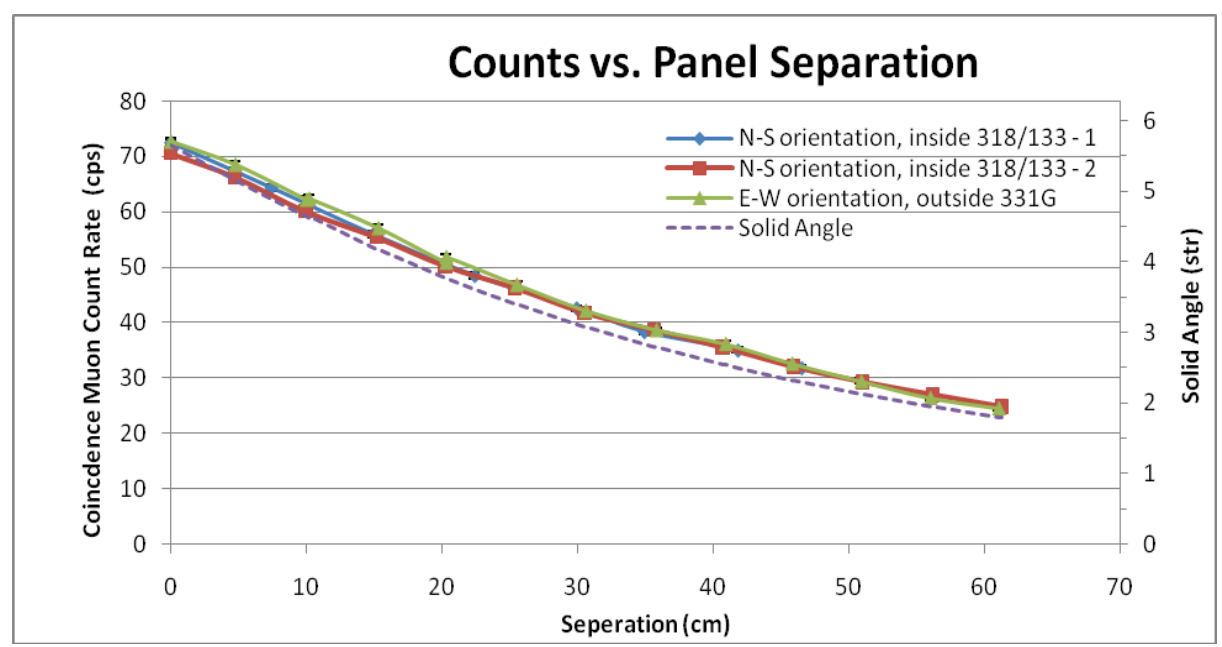

Figure 13 - Displays data and solid angle over a range of PVT panel separations. The error bars are too small to show up clearly on the plots.

Data collected at the two sites were found to be consistent. Since the indoor facility was a typical government/industrial lab at ground level, shielding was expected to minimally affect muon propagation compared to the outdoor site. No clear variation due to shielding difference was observed.

While the geomagnetic field does have east-west and north-south asymmetries, the panel area and solid angles are not influenced by this disparity. Data taken in each cardinal orientation were not observed to be significantly different. As discussed in section 2.2.2, altering the panel separation directly 
impacts the solid angle. The muon fluence tracks well with the solid angle at smaller angles, but the count rates rise to up to $\sim 8 \%$ above the theoretical curve at higher angles in Figure 13.

Assuming an isotropic muon flux, the coincidence rate should scale linearly with the solid angle subtended by the detector array (Figure 14).

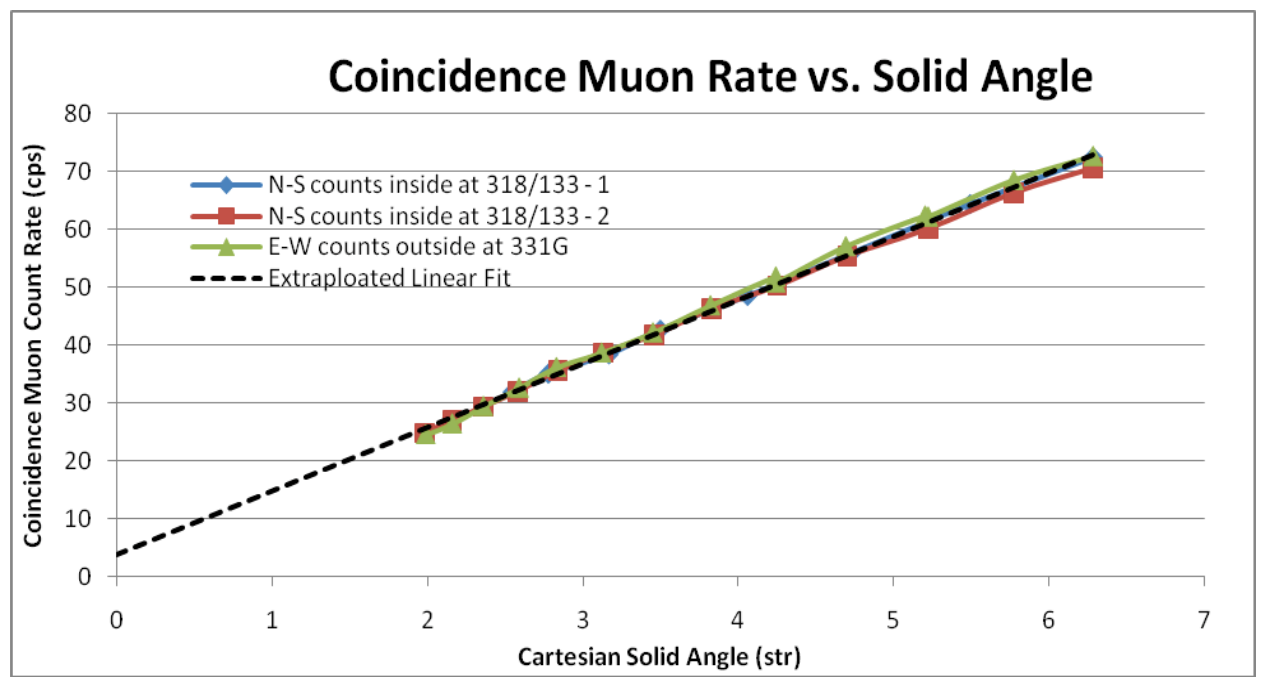

Figure 14 - As solid angle decreases with increasing panel separation, the muon count rate decreases. Accidental coincidences were subtracted from the muon count rate. The dashed line is an extrapolated linear fit giving a y-intercept of $3.7 \mathrm{cps}$.

Results from the two indoor experiments, with panels aligned in a north-south orientation, gave consistent results as shown in Figure 14. Statistical error bars are again on the order of $1 \%$, about the size of the plot symbols. These runs were also similar to data collected outdoors in an east-west orientation at low solid angles. For larger solid angles, the outdoors, east-west alignment yielded muon fluence rates $<3 \%$ higher than in the indoors, north-south scenario. Because there are two different variables in these measurements, indoors/outdoors and north-south/east-west, it is not possible to conclude which change caused this difference. Although the indoor facility was a single-level, typical industrial/government building, unlikely to measurably attenuate the muon signature, it may cause attenuation that would explain the indoor scenario registering fewer counts.

In both locations and orientations the muon fluence drops $\sim<3 \%$ lower than the linear fit at high solid angles. This could be a manifestation of an anisotropic muon flux, with the count rate decreasing at higher zenith angles [Grieder (2001)]. At higher angle angles, the subtended angle extends closer to the horizon. A decreased muon flux near the horizon is consistent with these results.

\subsection{Effects of Scintillation Orientation Testing on Muon Rates}

Measurements varying the orientation of the PVT scintillators were taken at three locations: indoors at 318/133; outdoors at $331 \mathrm{G}$ and underground at the 3425 Deep Lab. The underground facility was measured to be $12.3 \mathrm{~m}$ (40.4 feet) below ground level (not including the berm), and reported to be a $\sim 30 \mathrm{~m}$ water equivalent (MWE) depth. Due to the increased overburden, which attenuates muons and yields a lower count rate, data was taken for 10-minute intervals. Above ground testing was conducted at ground level at 318/133 and $331 \mathrm{G}$. At each site, the scintillators were spaced $40.8 \mathrm{~cm}$ apart and 
configured in a north-south and an east-west fashion. The data collected through these tests can be seen in Figures 15-21.

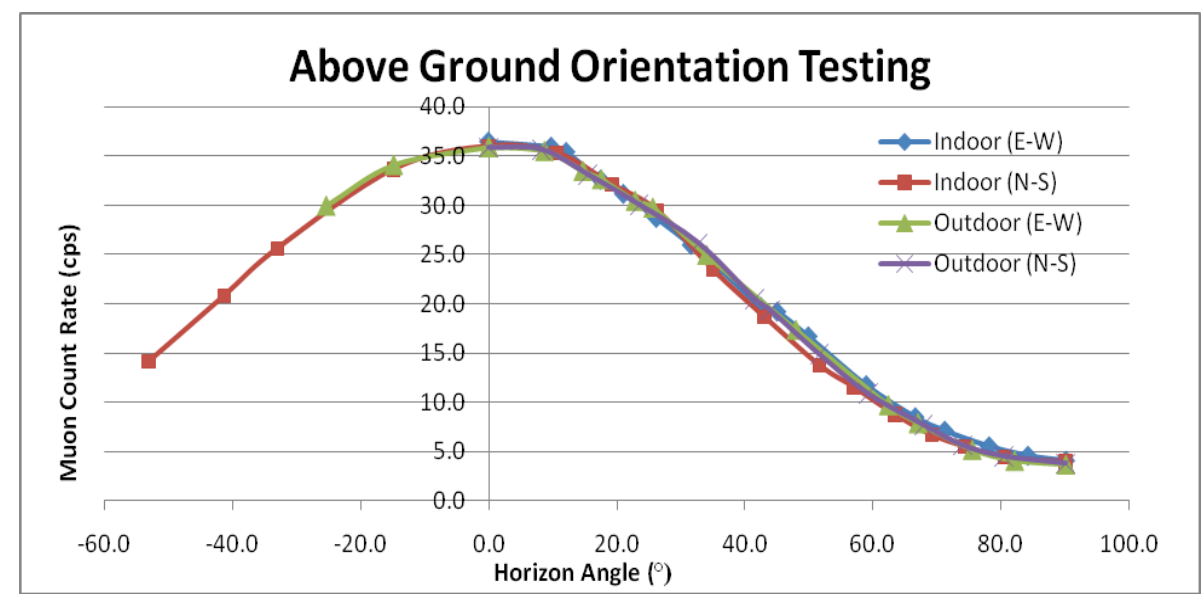

Figure 15 - Data collected at the two above ground locations, with orientations in both north-south and east-west alignments. The error bars are approximately the same size as the data point markers.

The collected data do not exhibit any significant difference between indoor and outdoor sites, in agreement with earlier measurements discussed in section 3.1. This is expected since the lab does not have significant shielding and thus does not measurably attenuate muons. Readings collected in the two cardinal orientations did not display any noticable differences. For two scenarios, limited data were obtained for inclinations rotated in the opposite direction, noted as negative angles in the Figures. These two data sets were consistent with each other, and within experimental error was symmetric about the $0^{\circ}$ vertical.

As discussed in section 2.2.2, at higher inclination angles portions of the detector solid angle are obscured by the horizon. Thus the measured angles plotted in Figure 15 differ from the actual effective solid angle. Corrections to the solid angle were made and are plotted in Figure 16.

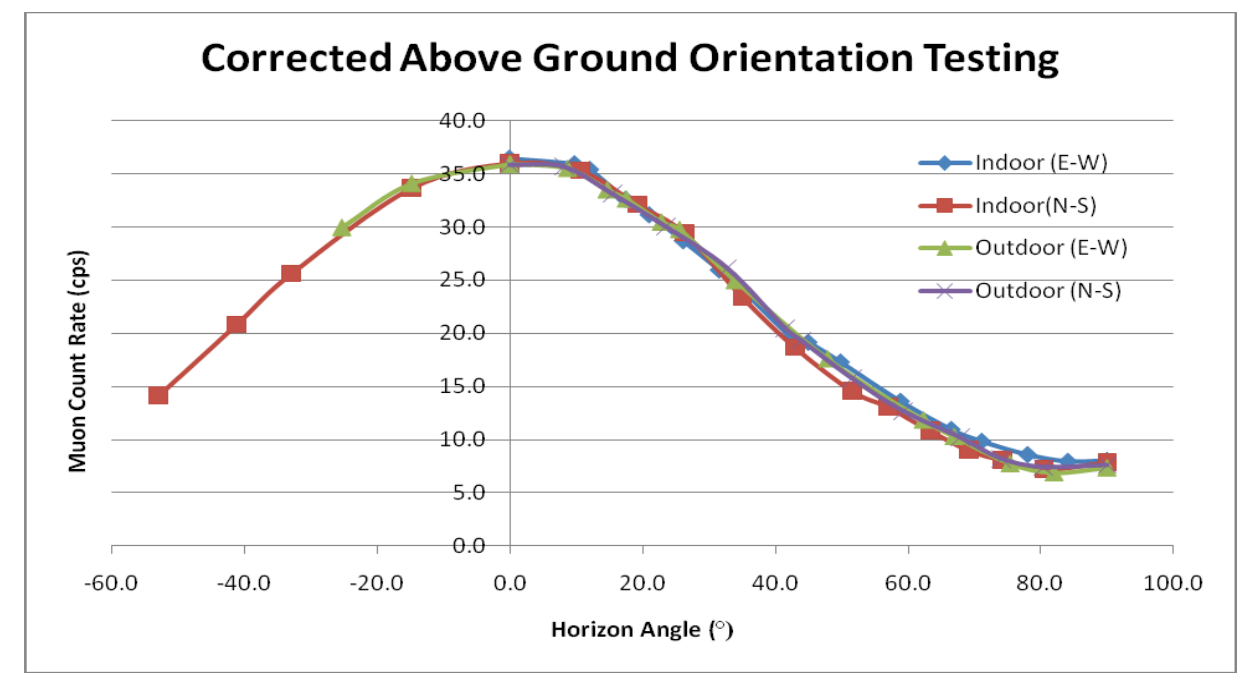

Figure 16 - Above ground data with the horizon angle corrected. The error bars were approximately the same size as the data point markers. 
Adjustments only needed to be made for angles above $46.7^{\circ}$. The conclusions drawn from Figure 15 still hold true for Figure 16. The only notable difference in Figure 16 is the appearance of a slight upturn for the vertical panel alignment at $90^{\circ}$. This could be due to the horizon correction being an overestimate. The simple geometric correction used to scale muon counts near the horizon assumes isotropic muon fluence above and below the horizon. However, the muon fluence near the horizon above ground and below ground is anisotropic, with the fluence being greater in the above ground scenario. Since the count rate for higher angles is scaled assuming muon isotropy near the horizon, the corrected count assumes the muon fluence below the horizon is the same as that above, thus making the corrected muon rate higher than the true value.

Data was compared to a similar study using PVT panels in coincidence at shallow depths [Lin et al. (2010)]. Lin used panels with a smaller area; their scaled data for two data sets are plotted with an uncorrected and corrected data set from the present work (Figure 17). Our data agree well with 'Case 1' from Lin, but are lower than his 'Case 2', a discrepancy that grows with increasing horizon angle.

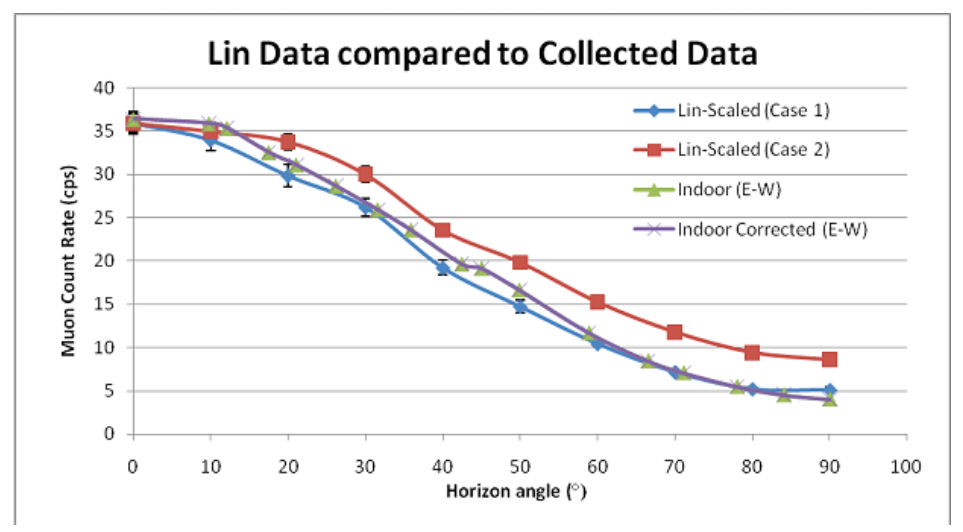

Figure 17 - Data from present study taken in an east-west alignment compared to scaled results from Lin, et al. The error bars were approximately the same size as the data point markers.

For measurements taken underground, the panels were laid parallel and separated by a fixed distance of $61.4 \mathrm{~cm}$. Eight runs were taken at various inclinations with the panels aligned north-south, and three east-west. The relationship between registered muon count rates and the angle with respect to the horizontal is shown in Figure 18.

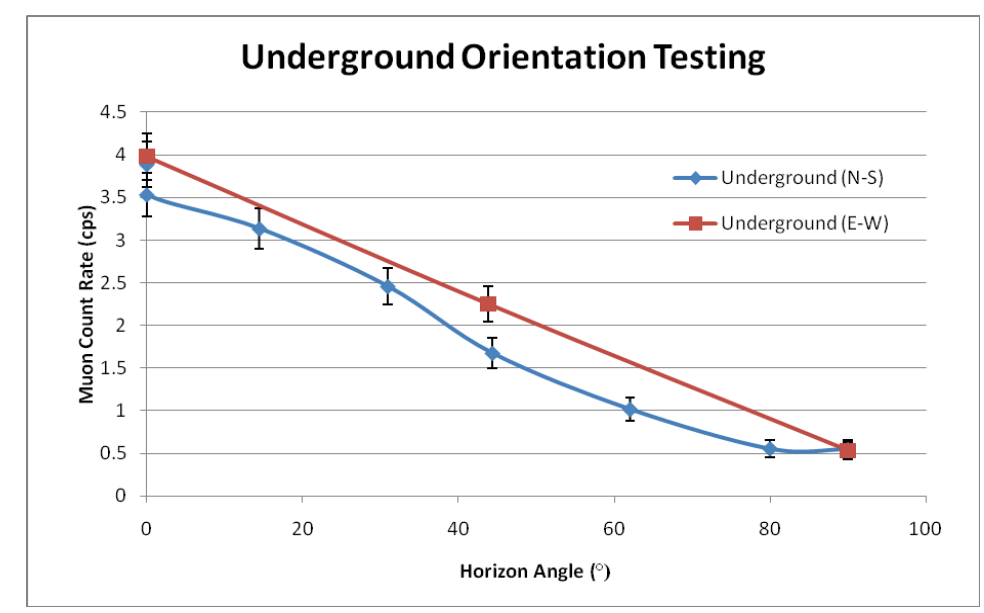

Figure 18 - North-south and east-west data with orientation testing completed underground. 
The north-south alignment has a lower count rate than the east-west at all angles tested. The design of the building features concrete entry structures on the east and west sides which prevent the inclusion of the berm that is present along the long north and south faces (see Figures 10, 23). This may lead to decreased shielding, and a corresponding increased count rate, in the east-west orientation.

Composite data, with uncorrected runs for all sites and for different cardinal orientations, is shown in Figure 20.

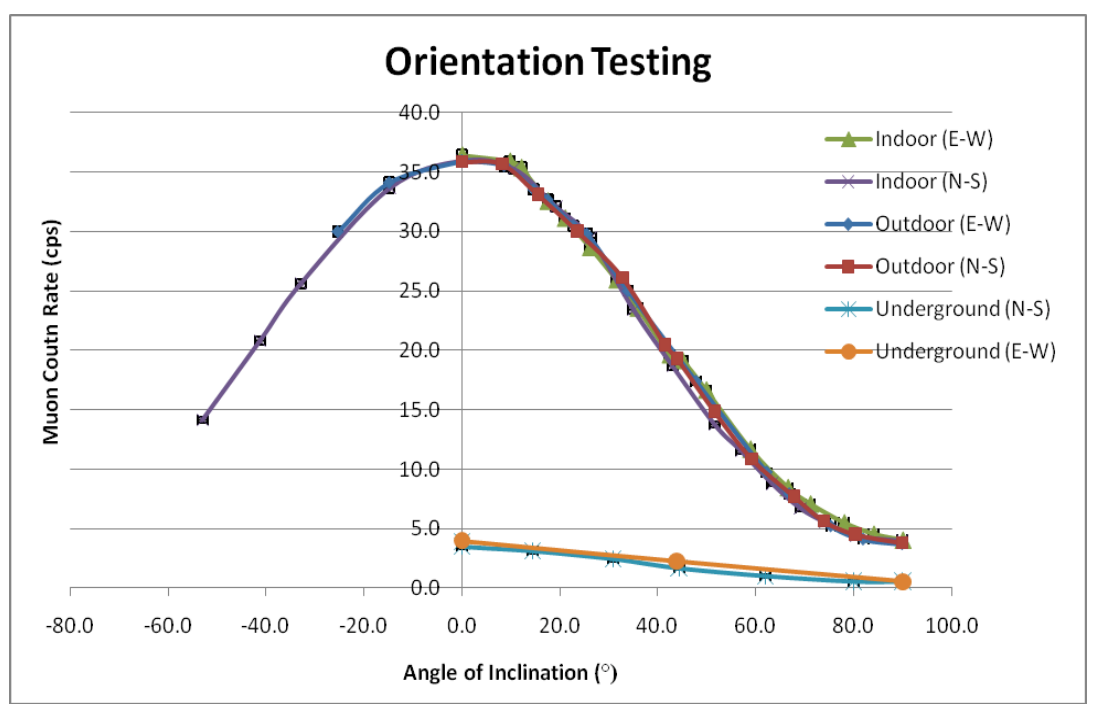

Figure 19 - Composite display of all uncorrected, with no horizon cutoff adjustment, data runs for varying inclinations above ground and underground. The error bars were approximately the same size as the data point markers.

Data from the underground and above ground measurements in Figure 20 follow the same general trend, with decreasing count rates as the detector angle with the horizontal increases. However the spectra from the underground measurements are more suppressed at lower angles than at higher angles compared to the above ground measurements, although the suppression ratios of the maxima at $0^{\circ}$ to the minima at $90^{\circ}$ are similar. For the above ground measurements the peak is $9.46 \pm 0.48$ times larger than the minimum, while for the underground measurements the peak is about $7.09 \pm 0.54$ times larger. Although these results do not agree within error, it is possible that these ratios would correspond if additional underground data were collected in the underground facility. However, assuming these results are accurate, one possible explanation for this discrepancy is that muons detected above ground only need to pass through the atmosphere ( $\sim 10 \mathrm{MWE})$ and so this spectrum contains relatively more low energy muons, while in the underground facility muons must travel through a greater thickness of material. Thus the lower energy muons are preferentially attenuated in the underground case and the remaining spectrum, composed of relatively higher energy muons, is less affected by the angle of inclination of the detector. This effect could preferentially impact muons incident at angles close to the horizon due to the muon anisotropy discussed earlier.

The difference between the above and below ground count rate is reduced as the inclination angle increases. This could be a result of the differing paths and energy spectra of the muons detected. Muons incident along trajectories closer to the horizon must travel a farther distance to reach the detector than muons entering vertically. Above ground this additional path is in air $\left(\sim 1 \mathrm{mg} / \mathrm{cm}^{2}\right)$, where the minimally ionizing muons lose $\sim 2 \mathrm{keV} / \mathrm{cm}$, while underground $\left(\sim 5 \mathrm{~g} / \mathrm{cm}^{2}\right)$, they lose $\sim 10 \mathrm{MeV} / \mathrm{cm}$. This means 
muons entering at $0^{\circ}$ have a lower average energy underground, thus many of the muons registered by the detector above ground are filtered out by the overburden and cannot reach the detector underground. Based on muons being minimally ionizing particles, and the underground facility being below ground $12.3 \mathrm{~m}$ (not including the berm), muons need to have an energy of $>\sim 12 \mathrm{GeV}$ to reach the underground facility. Above ground this threshold, for the $10 \mathrm{MWE}$ atmosphere, is $\sim 2 \mathrm{GeV}$. Because the muons entering closer to horizontal at the surface or underground must on average of relatively higher energy muons than those entering vertically, such muons are more likely to be $>40 \mathrm{GeV}$, and less likely to be shielded by the overburden. In other words, if a muon has enough energy to enter horizontally at the surface, then it is more likely to have the energy to enter horizontally underground as well.

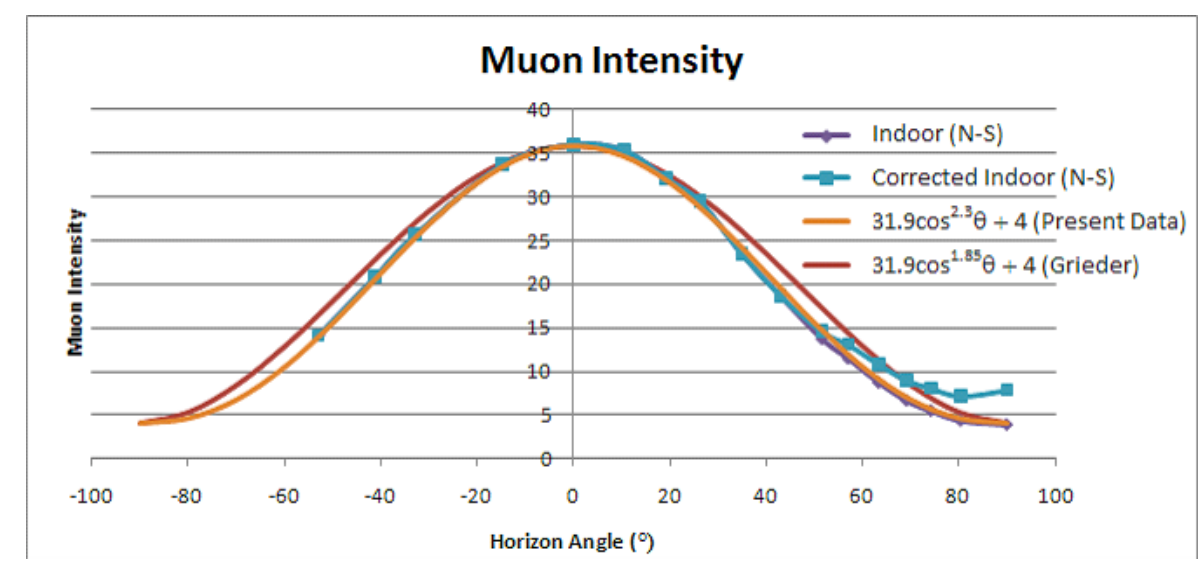

Figure 20 - Comparison of experimental data to that of cosine curves were placed together to compare our indoor data to Grieder's data.

Several authors [Lin (2010); Grieder (2001); Dragić (2008)] have reported on the results of fitting a $I(\theta)=I_{0} \cos ^{n} \theta$ curve to experimental findings. Grieder recommends a value of $n=1.85 \pm 0.10$ [Grieder (2001)], while Lin reports finding $n=1.21$ and 1.74 [Lin (2010)]. Data from this study, along with Grieder's advised value, are plotted in Figure 21. Our results match Grieder's fit with a value of $n=$ 2.3, higher than reported by others. This could be a result of the literature focus on muon fluence at larger depths. At shallow depths lower muon energies are preferentially attenuated. For instance, as noted in above, at a depth of $12 \mathrm{~m}$ minimally ionizing muons lose $12 \mathrm{GeV}$. At a depth of $2 \mathrm{~km}$, such as the IceCube experiment, this cutoff increases to $2000 \mathrm{GeV}$, more uniformly suppressing the muon fluence at all angles. By this argument, at shallower depths the cosine exponent $n$ would increase, consistent with our findings.

\subsection{Depth Attenuation of Muons}

This work in the 3425 Deep Lab adds to measurements taken with the detector horizontally aligned $\left(0^{\circ}\right)$ in August 2009 and reported in PNNL-18855 [Kouzes et al. (2009)]. That data was collected with a single $10 \mathrm{~cm} \times 10 \mathrm{~cm} \times 40 \mathrm{~cm} \mathrm{NaI(Tl)} \log$ at a time when the overburden was in place, but the overlaying berm had not yet been completed (Figure 22). The muon data in that case was taken with a low gain and low voltage in order to have the minimum ionizing signal of $\sim 75 \mathrm{MeV}$ appear on the spectra. In the present case using the $100 \mathrm{~cm} \times 40 \mathrm{~cm} \times 5 \mathrm{~cm}$ PVT panels where the muons had a minimum ionizing signal of $\sim 10 \mathrm{MeV}$, data was collected recording both individual panel counts and 
counts in coincidence. The site, with the completed berm, is shown in Figure 23. Data was collected over various lengths of time, and so was scaled to 20 -minute runs for comparative purposes in Table 1. The right hand column shows the ratio of muon fluence at depth to that at the surface.

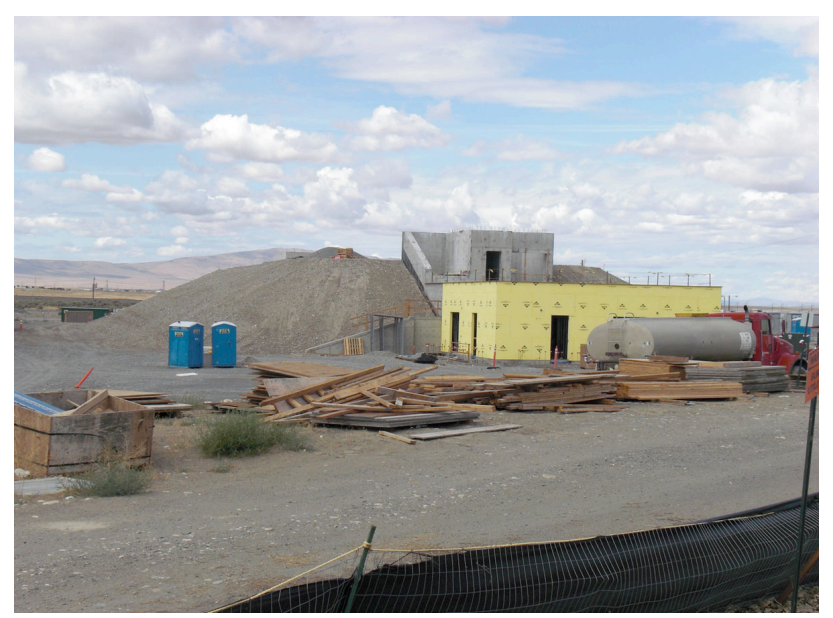

Figure 21 - Surface earthen berm from southeast of Building 3425 in August 2009 during construction.

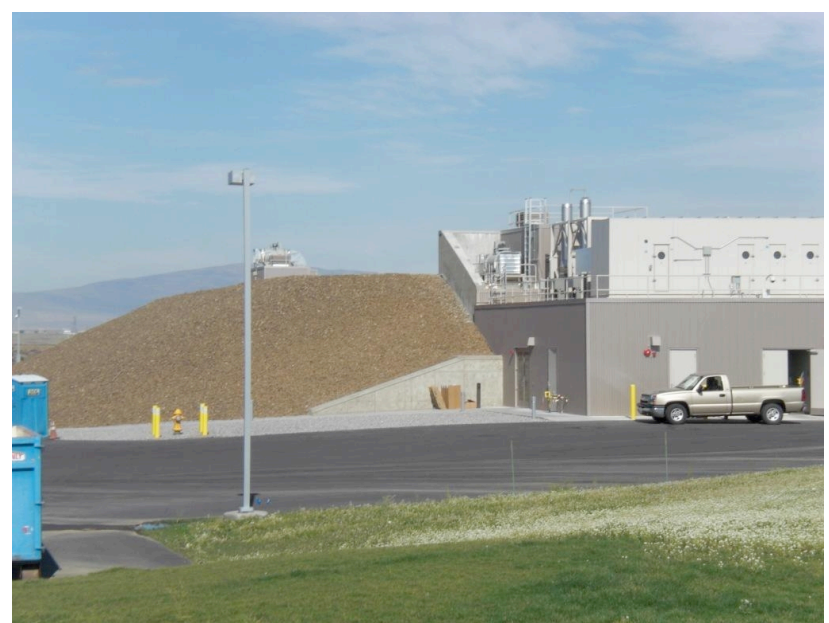

Figure 22 - Surface berm of Building 3425 in July 2010 at completion of construction. Note additional rock cap earthen bunker compared to Figure 22.

Table 1: Summary of data comparing muon fluence at surface to that in the 3425 Deep Lab

\begin{tabular}{|l|l|l|l|l|l|}
\hline & Detector used & $\begin{array}{l}\text { Collection } \\
\text { times }\end{array}$ & $\begin{array}{l}\text { Counts at } \\
\text { surface (scaled } \\
\text { to 20-min) }\end{array}$ & $\begin{array}{l}\text { Counts in Deep } \\
\text { Lab (scaled to } \\
\text { 20-min) }\end{array}$ & $\begin{array}{l}\text { Ratio Deep } \\
\text { Lab to surface }\end{array}$ \\
\hline August 2009 & $\begin{array}{l}1-4 \text { "x4"x16" } \\
\text { NaI(T1) log }\end{array}$ & 20 min & 3795 & 532 & 0.140 \\
\hline July 2010 & $\begin{array}{l}2- \\
5 \mathrm{~cm} 440 \mathrm{~cm} x 120 \mathrm{~cm} \\
\text { PVT panels in } \\
\text { coincidence }\end{array}$ & $\begin{array}{l}3 \text { min } \\
\text { (surface) or 10 } \\
\text { min (depth) }\end{array}$ & 43220 & 4562 & 0.106 \\
\hline
\end{tabular}


The Deep Lab was measured to be roughly $12.3 \mathrm{~m}$ below the surface, not including the berm. In general, numerical models for attenuation of muon intensity with depth relate to neutrino detection efforts, and are therefore intended for much greater depths. To estimate the equivalent water depth of the 3425 Deep Lab two formulae for intensity as a function of depth were used. The first, developed for greater depths, is given by

$$
I(X)=A_{o} \frac{X^{-\alpha} e^{-\beta x}}{(X+H)}
$$

where $A_{o}=7.5 \mathrm{hg} / \mathrm{cm}^{2}, \alpha=1.13, h_{l}=35.0 \mathrm{hg} / \mathrm{cm}^{2}$ and $\beta=5.5 \times 10^{-4} \mathrm{~cm}^{2} / \mathrm{hg}$ [Miyake (1963)]. The second, altered to apply for shallow depths, is

$$
I(X)=\frac{K e^{-\beta x}}{\left(X^{\alpha}+a\right)(X+H)}
$$

where $K=270.7 \mathrm{hg} / \mathrm{cm}^{2}, a=75, H=200 \mathrm{hg} / \mathrm{cm}^{2}, \alpha=1.68, \beta=5.5 \times 10^{-4} \mathrm{~cm}^{2} / \mathrm{hg}$, where $X$ and $I$ have the same units as above [Barbouti et al. (1983)]. The units $\mathrm{hg} / \mathrm{cm}^{2}$ (hg is hectograms) are approximately the same as meters water equivalent (MWE). The depth is measured from the top of the atmosphere, and sea level is at a depth of $\sim 1000 \mathrm{~g} / \mathrm{cm}^{2}\left(10 \mathrm{hg} / \mathrm{cm}^{2}\right)$.

Figure 24 includes data from this work, and well as from prior studies at shallow depth [Crookes et al. (1972); Percy et al. (1977)]. Both data sets from this study are consistent with the prior studies cited. The dashed line in Figure 24 represents the numerical fit using the above formula. The atmosphere is taken to be equivalent to an attenuation water depth of $10 \mathrm{MWE}$. Using this fit, the 2009 data indicates a Deep Lab depth relative to the top of the atmosphere of $37 \mathrm{MWE}$, or $27 \mathrm{MWE}$ relative to the surface using the Miyake fit, and a depth relative to the top of the atmosphere of $47 \mathrm{MWE}$, or $37 \mathrm{MWE}$ relative to the surface using the Barbouti fit. The present data yields a depth relative to the top of the atmosphere of $44 \mathrm{MWE}$, or $34 \mathrm{MWE}$ relative to the surface using Miyake, and a depth relative to the surface of 55 MWE, or 45 MWE relative to the surface using Barbouti.

Since the current data contained some gamma ray counts, which would tend to inflate these estimates, these markers should be taken as high-end limits. While the Miyake fit was developed to apply for depths near sea level to depths $\sim 5 \mathrm{~km}$, published comparative data is given for depths in the range of $70 \mathrm{~m}$ to $5000 \mathrm{~m}$ [Wright (1974); Davitaev et al.(1969); Higashi et al. (1968)]. The Miyake formula correlates well over this region, but there is only one experimental data point presented for depths less than $100 \mathrm{~m}$. This data is consistent with both the 3425 building contractor's claim of a depth of at least $30 \mathrm{MWE}$, and with some additional material, namely the berm and north and south-facing concrete structures, having been added during final construction between 2009 and 2010 . 
PNNL- 19632

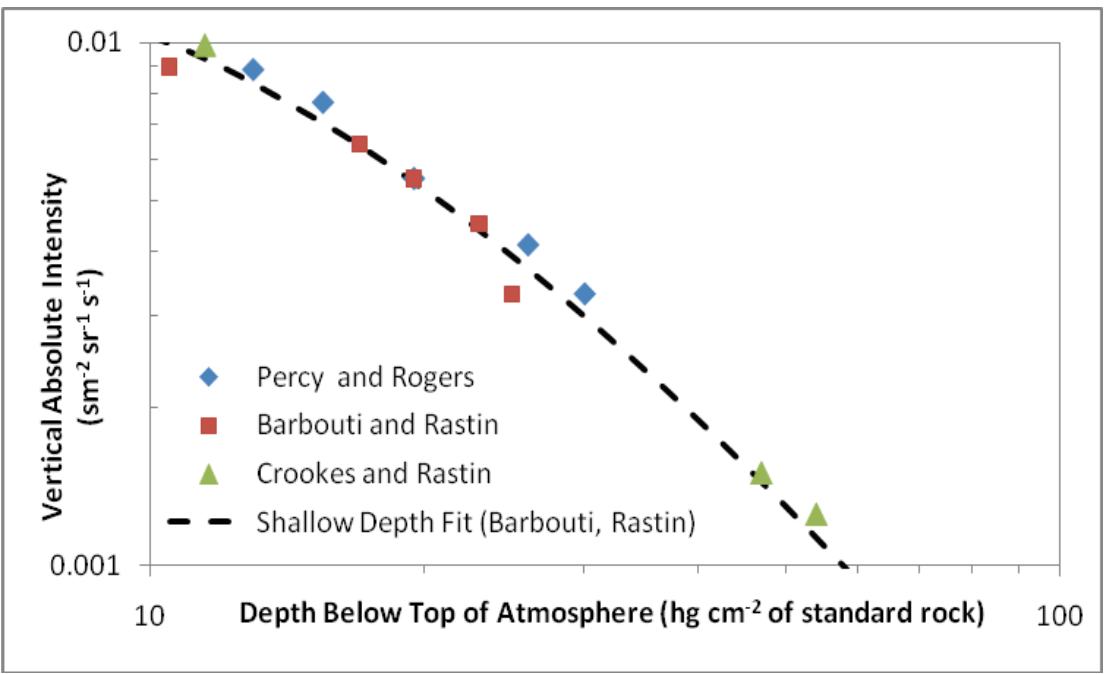

Figure 23 -Data from several studies of muon attenuation at shallow depth. The fit from a numerical model developed by Barbouti et al. for shallow depths is given by the dotted line [Barbouti et al. (1983)].

Results from a study done conducted in a shallow underground cavern by Effremenko are shown in Figure 25 [Effremenko (2010)].

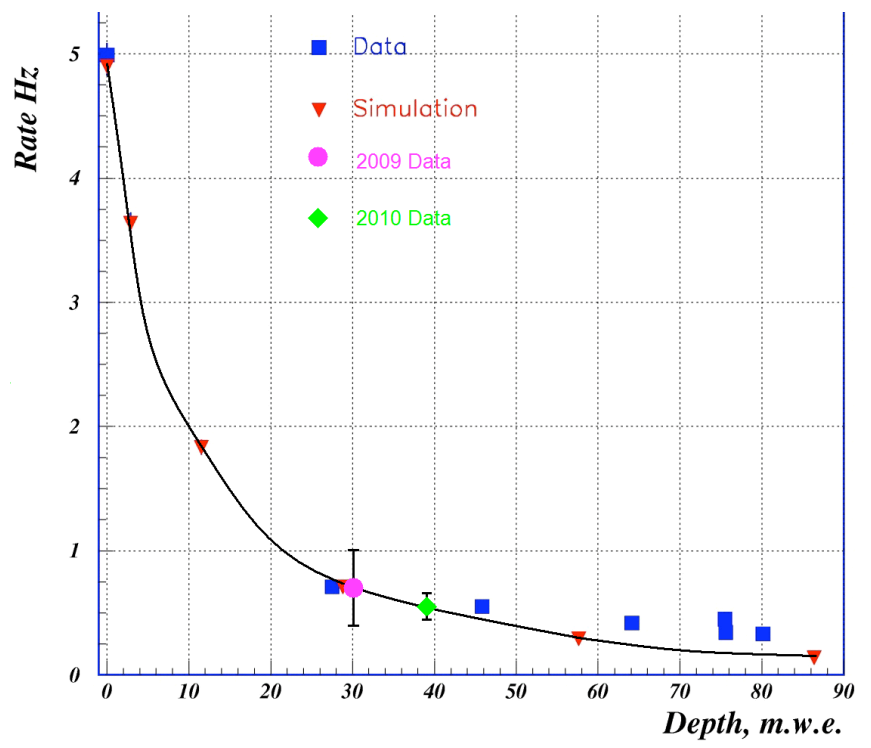

Figure 24 - Data presented by Effremenko, along with data collected at the underground lab in 3425 during August 2009 and July 2010. The solid line is the best fit for data generated by a GEANT simulation performed by Effremenko. August 2009 error bars are larger due to poorer statistics relative to the July 2010 data.

Effremenko collected a series of data points at various depths and conducted a GEANT simulation to model the results. The solid line in Figure 25 is our best fit to that GEANT model. Data from Table 1, expressed as the ratio of surface counts to those at depth, are shown. The August 2009 study yields a depth of $35 \pm 15$ MWE relative to the surface based on this comparison, while the current work gives a depth of $38 \pm 8 \mathrm{MWE}$ relative to the surface. The error for the 2009 data is relatively greater due to lower statistics compared to the present work coupled with the decreasing differential muon 
attenuation at higher depths. The slightly higher value for the present data is consistent with additional construction including the deposition of a layer of rip rap on the berm and the installation of the concrete entry structures.

\subsection{Diurnal Dependence of Muon Rates}

Measurements for diurnal testing were conducted indoors at 318/133 in a north-south configuration. Data was acquired over a seven-day period, and parceled in one-hour bundles yielding 168 data sets. The results for the count rate are shown in Figure 26.

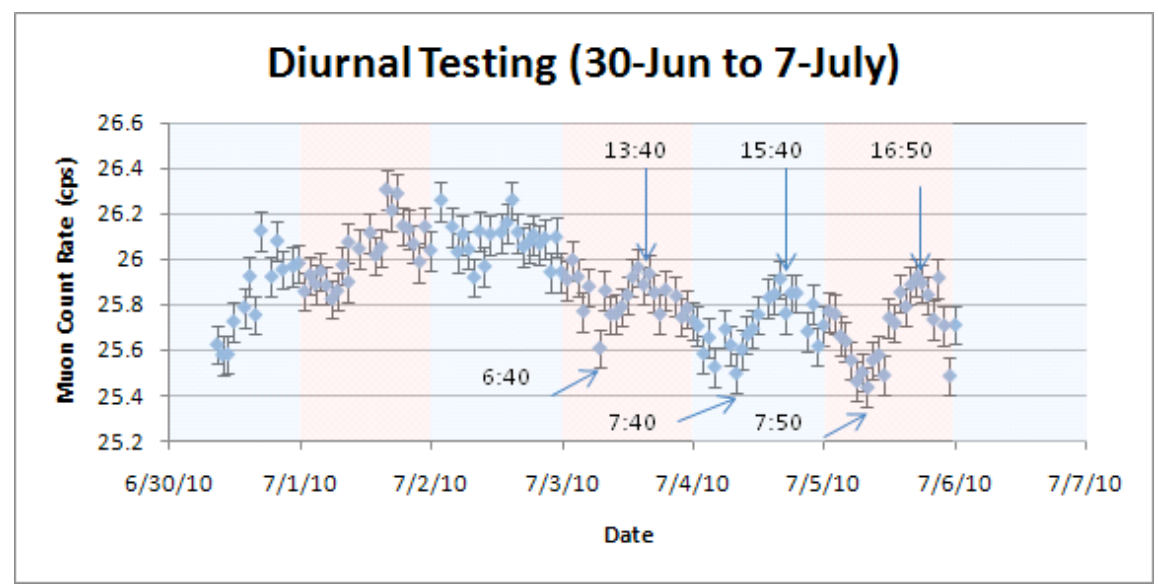

Figure 25 - The muon count rate over an eight day period. The shaded red and blue regions denote 24hour intervals. The approximate times for daily minima and maxima in the periodic phase are indicated.

The results from the diurnal testing show two distinct regions. The initial four days exhibited some periodicity, however this was shrouded by some overlaying noise. The final three days display a much clearer harmonic behavior with $\sim 25$-hour period. This is a reflection of the 24-hour rotation period of the earth, coupled with the 27-day rotation period of the sun. The latter introduces roughly an additional hour for a point on the earth to come back into phase with an identical point on the sun. The aperiodic behavior could be attributed to a period of high solar activity, while the periodic behavior could be a manifestation of more stable solar activity combined with the phase periodicity of the earth-sun system. 


\section{Conclusion}

A series of experiments were undertaken utilizing two large $(100 \mathrm{~cm} \times 40 \mathrm{~cm} \times 5 \mathrm{~cm})$ PVT detectors in coincidence to investigate muon flux dependence on an array of parameters including variations in solid angle subtended by the detector, the detector inclination with the horizontal, the depth underground, and diurnal effects. These tests were conducted inside at Building 318/133, outdoors at Building 331G, and underground at Building 3425. Results of the analysis elucidated muon behavior for all tests.

The muon flux in above ground locations increased linearly as the solid angle subtended by the detector increased. Data was consistent for both the indoor and outdoor sites, and showed little dependence on cardinal orientation. Some small deviations from theoretical models were observed for larger solid angles, which were attributable to a decrease in the muon flux for increasing zenith angles.

Muon fluence followed a $\cos ^{2.3} \theta$ correlation when the detector inclination was varied, where $\theta$ is defined with respect to the vertical. This exponential dependence was larger than recommended or reported by other researchers [Grieder (2001); Lin et al. (2010)], however such results are given for depths on the order of $\mathrm{km}$. For this study, conducted at a shallow depth, the lower exponent is thought to be reflective of the preferential attenuation at greater depths of muons with larger energies relative to those detected near the surface.

The muon attenuation underground, measured at one depth at the underground lab at Building 3425, was shown to be consistent with earlier measurements and prior studies at similar depths. A formula for muon attenuation at shallow depths [Barbouti et al. (1983)] yielded depths of 27 MWE (2009 study) and $37 \mathrm{MWE}$ (present study) relative to the surface. A second method was employed to estimate the depth by comparing present results to a study by Effremenko at shallow depths, which included a GEANT simulation [Effremenko (2010)]. Utilizing a best-fit curve for the simulation data, depths of $35 \pm 15$ MWE (2009 study) and $38 \pm 8$ MWE (present work) relative to the surface were obtained. These data are consistent, and a slightly greater depth for the present study is consistent with the addition of surface material during the final phases of construction.

The diurnal behavior of the muon flux exhibited both periodic and aperiodic intervals, each on the order of days, for data collected over a 7 -day period. The harmonic phase exhibited a $\sim 25$-hour period, reflecting the 24-hour rotation of the earth coupled with the 1/27 rotation of the sun in one day. Maxima occurred at $\sim 3$ pm PST and minima at $\sim 8$ am PST for the timeframe observed.

Additional measurements could be taken for the separation testing to investigate which parameter (inside/outside or north-south/east-west) was responsible for the increase in the muon count rate. Further inclination measurements at various shallow depths could reveal the variation of the exponent $n$, used in the numerical $\cos ^{2} \theta$ model, with depth. Additional tests in the underground facility, particularly for the east-west orientation, would provide a more complete set of data points and allow a more robust examination of the lower muon flux in the north-south orientation. A detailed comparison of solar activity over the timeframe for which diurnal data was collected would be useful in establishing a possible causal relationship. Further tests for an extended timeframe would better reveal the extent of the periodic and aperiodic intervals observed, and also provide the opportunity to more strongly establish a relationship between the diurnal period with that of the $\sim 25$-hour orbital resonance of the earth-sun system. 


\section{References}

1) Barbouti AI and Rastin BC. 1983. "A study of the absolute intensity of muons at sea level and under various thicknesses of absorber." J. Phys. G: Nucl. Phys. 9 (1983) 1577-1595.

2) Bogdanova LN, Gavrilov MG, Kornoukhov VN, and Starostin AS. 2006. "Cosmic muon flux at shallow depths underground." Phys.Atom. Nucl. 69 (2006) 1293-1298 arXiv:nuclex/0601019v1.

3) Crookes JN and Rastin BC. 1972. "An investigation of the absolute intensity of muons at sealevel.” Nucl. Phys. B 39493

4) Davitaev LN, Fedorov WM, Trubkin YA and Vavilov YN. 1969. Proceedings of the $11^{\text {th }}$ International Conference on Cosmic Rays, Budapest, 4, 53 (1969)

5) Effremenko Y (2010). "Muon Flux at the Cherokee Caverns". Majorana conference, personal communication with Kouzes RT

6) Ely JH, Shepard CL, and Siciliano ER. 2004. "Energy Calibration of PVT Detectors.” APS April Meeting.

7) Grieder PKF. 2001. “Cosmic Rays at Earth.” Elsevier.

8) Haymes RC. 1965. “Terrestrial and solar neutrons.” Rev. Geophys., 3(3), 345-364, doi:10.1029/RG003i003p00345.

9) Higashi S. et al. 1966. Nuovo Cimento 47786 (1966).

10) Knoll GF. 2000. "Radiation Detection and Measurement $3^{\text {rd }}$ Ed." John Wiley and Sons, New York.

11) Kouzes RT. 2009. "The ${ }^{3}$ He Supply Problem.” Pacific Northwest National Laboratory White Paper PNNL-18388.

12) Kouzes RT, Ely JH, Seifert A, Siciliano ER, Weier DR, and Windsor LK. 2008a. "Cosmic-rayinduced ship-effect neutron measurements and implications for cargo scanning at borders." NIM-A, 587:89-100.

13) Kouzes RT, Siciliano ER, Ely JH, Keller PE, and McConn, Jr RJ. 2008. "Passive Neutron Detection for Interdiction of Nuclear Material at Borders, " Nuclear Instruments and Methods in Physics Research A vol. 584, pp. 383-400(2008b), PNNL-SA-54753.)

14) Lin JW et al. "Measurement of angular distribution of cosmic-ray muon fluence rate." Nuclear Instruments \& Methods in Physics Research Section A 619, no. 1-3 (July 2010): 24-27. Academic Search Premier, EBSCOhost (accessed July 13, 2010).

15) Mailyan B and Chilingarian A. 2010. "Investigation of diurnal variations of cosmic ray fluxes measured with using ASEC and NMDB monitors." Advances in Space Research 45, no. 11 (June 2010): 1380-1387. Academic Search Premier, EBSCOhost (accessed July 13, 2010).

16) McNally F. “Simulating Light Production in IceTop.” Carleton College (accessed July 172010 ). [Figure 3.]

17) Percy JC and Rogers IW. 1977. "Cosmic ray intensities at shallow depths" Proc. 15th Int. Conf on Cosmic Rays, Volume 6. (1977) 99-103.

18) Pevey, RE. "Lesson 2: Directions and Solid Angles." NE 406 Radiation Protection and Shielding. 1998. University of Tennessee. 27 Jul. $2010<$ http://web.utk.edu/ rpevey/NE406/lesson2.htm>. 
19) Thompson J. "Solar diurnal variation of cosmic-ray intensity as a function of latitude." Phys. Rev. 54. (1938) 93-96.

20) Tiwari CM and Tiwari DP. 2008. "Characteristics of high energy cosmic ray diurnal anisotropy on day-to-day basis." Cosmic Research 46, no. 5 (September 2008): 465-468. Academic Search Premier, EBSCOhost (accessed July 14, 2010).

21) Wolfram Research, Inc. 2008. “Mathematica Edition: Version 7.0.” Wolfram Research, Inc. Champaign Illinois. 2008.

22) Wright AG. 1974. "A study of muons underground and their energy spectrum at sea level." J. Phys. A: Math., Nucl. Gen.. Vol. 7, No. 16, 1974. 
PNNL- 19632

\section{Bibliography}

*-denotes works cited in the references

1) Andreotti E, et al. 2010. "Muon-induced backgrounds in the CUORICINO experiment." Astroparticle Physics 34, no. 1 (August 2010): 18-24. Academic Search Premier, EBSCOhost (accessed July 13, 2010).

2) Badhwar GD, Deney CL, Dennis BR, and Kaplon MF. 1967. "Measurements of the Low-Energy Cosmic Radiation during the Summer of 1966.” Physical Review, vol. 163, Issue 5 (1967) 13271342

3) Banjanac R, et al. 2007. "Cosmic-ray Muon Flux In Belgrade." AIP Conference Proceedings 899, no. 1 (April 23, 2007): 543. Academic Search Premier, EBSCOhost (accessed July 13, 2010).

4) *Barbouti AI and Rastin BC. 1983. "A study of the absolute intensity of muons at sea level and under various thicknesses of absorber." J. Phys. G: Nucl. Phys. 9 (1983) 1577-1595.

5) Belovt V, Bieber JW, Eroshenkot EA, Evenson P, Pyle R, and Yanke VG. 2003. "Cosmic Ray Anisotropy Before and During the Passage of Major Solar Wind Disturbances." Adv. Space Res. Vol. 31, No. 4, 919-924 (2003) doi: 1O.1016/SO273-1177(02)00803-7

6) *Bogdanova LN, Gavrilov MG, Kornoukhov VN, and Starostin AS. 2006. "Cosmic muon flux at shallow depths underground." Phys.Atom. Nucl. 69 (2006) 1293-1298 arXiv:nuclex/0601019v1.

7) Bugaev EV, Misaki A, Naumov VA, Sinegovskaya TS, Sinegovsky SI, Takahashi N, 1998. “Atmospheric Muon Flux at Sea Level, Underground, and Underwater." Physical Review D, Volume 58, 054001.

8) Casimiro E, Simã FRA, Anjos JC, 2007. "Estimate of Cosmic Muon Background for Shallow Underground Neutrino Detectors." $30^{\text {th }}$ International Cosmic Ray Conference.

9) *Crookes JN and Rastin BC. 1972. "An investigation of the absolute intensity of muons at sealevel.” Nucl. Phys. B 39493

10) *Davitaev LN, Fedorov WM, Trubkin YA and Vavilov YN. 1969. Proceedings of the $11^{\text {th }}$ International Conference on Cosmic Rays, Budapest, 4, 53 (1969)

11) Dragić A, Joković D, Banjanac R, Udovičić V, Panić B, Puzović J, Aničin I, 2008. "Measurement of Cosmic Ray Muon Flux in the Belgrade Ground Level and Underground Laboratories.” Nuclear Instruments and Methods in Physics Research A 591 (2008) 470-475.

12) *Effremenko Y (2010) - "Muon Flux at the Cherokee Caverns". Majorana conference, personal communication with DKouzes

13) *Ely JH, Shepard CL, Siciliano ER. 2004. “Energy Calibration of PVT Detectors.” APS April Meeting.

14) Fenyves EJ, Cherry ML, Deakyne M, Lande K, Lee CK, and Steinberg RI. 1981. "Angular distribution of comic-ray muons at $4200 \mathrm{~m}$. w. e." Proceedings of the $17^{\text {th }}$ International Cosmic Ray Conference. Vol. 10 (1981): 317. 
15) Garyaka AP, et al. 2009. "Primary cosmic ray energy spectrum in terms of the GAMMA muon data." Nuclear Physics B Proceedings Supplement 196, (December 2009): 387-390. Academic Search Premier, EBSCOhost (accessed July 13, 2010).

16) *Grieder PKF. 2001. “Cosmic Rays at Earth.” Elsevier.

17) Groom DE, Mokhov NV, Striganov, SI, 2001. "Muon Stopping Power and Range Tables: 10 MeV-100 TeV." Atomic Data and Nuclear Data Tables, Vol. 76, No. 2.

18) Harley JH (Chair), et al. 1987. NCRP, "Exposure of the population in the United States and Canada from natural background radiation", NCRP Report No.94, 1987.

19) *Haymes RC. 1965. “Terrestrial and solar neutrons.”, Rev. Geophys., 3(3), 345-364, doi:10.1029/RG003i003p00345.

20) *Higashi S. et al. 1966. Nuovo Cimento 47786 (1966).

21) Hurwitz M, Pilcher J, 2004. "Simulation of Cosmic Ray Muon Flux at Shallow Depths Underground. ”July 22, 2010. mwtheta13.uchicago.edu/text/Muon.pdf

22) Kempa J and Krawczynska A. 2007. "Muons in the Cosmic Radiation." $30^{\text {th }}$ International Cosmic Ray Conference, Mérida, Mexico, 2007.

23) Kitamura T. 1975. "Muons and Neutrinos in the Cosmic Radiation." $14^{\text {th }}$ International Cosmic Ray Conference, Munich, Germany, 1975.

24) Klein SR. 2009. "Muon Production in Relativistic Cosmic-Ray Interactions." Nuclear Physics A 830, no. 1-4 (November 2009): 869c-872c. Academic Search Premier, EBSCOhost (accessed July 13, 2010).

25) *Knoll GF. 2002. Radiation Detection and Measurement $3^{\text {rd }}$ Ed. John Wiley and Sons, New York.

26) *Kouzes RT. 2009. “The ${ }^{3}$ He Supply Problem.” Pacific Northwest National Laboratory White Paper PNNL-18388.

27) *Kouzes RT, Ely JH, Seifert A, Siciliano ER, Weier DR, and Windsor LK. 2008. "Cosmic-rayinduced ship-effect neutron measurements and implications for cargo scanning at borders." NIM-A, 587:89-100.

28) *Kouzes RT, Siciliano ER, Ely JH, Keller PE, and McConn, Jr RJ. 2008. "Passive Neutron Detection for Interdiction of Nuclear Material at Borders." Nuclear Instruments and Methods in Physics Research A vol. 584, pp. 383-400(2008), PNNL-SA-54753.)

29) Kozyarivsky V and Lidvansky A. 2008. "On the magnitude and direction of the anisotropy of galactic cosmic rays." Astronomy Letters 34, no. 2 (February 2008): 113-117. Academic Search Premier, EBSCOhost (accessed July 13, 2010).

30) Kudela K, Firoz KA, Langer R, Kollár V, “On Diurnal Variation of Cosmic Rays: Statistical Study of Neutron Monitor Data Including Lomnický Štit. July 21, 2010. ikfia.ysn.ru/pdf/Cosmic_Ray_Symp/s4.15.pdf

31) Kudela K and Storinib M. 2005. "Cosmic ray variability and geomagnetic activity: A statistical study. ”Journal of Atmospheric and Solar-Terrestrial Physics, 67(10), (July 2005): 907-912. doi:10.1016/j.jastp.2005.02.018. 
32) *Lin JW et al. 2010. "Measurement of angular distribution of cosmic-ray muon fluence rate." Nuclear Instruments \& Methods in Physics Research Section A 619, no. 1-3 (July 2010): 24-27. Academic Search Premier, EBSCOhost (accessed July 13, 2010).

33) M uuml hry H and Ritter P. 2002. "Muons in the Classroom." Physics Teacher 40, no. 5 (May 2002): 294. Academic Search Premier, EBSCOhost (accessed July 13, 2010).

34) *Mailyan B and Chilingarian A. 2010. "Investigation of diurnal variations of cosmic ray fluxes measured with using ASEC and NMDB monitors." Advances in Space Research 45, no. 11 (June 2010): 1380-1387. Academic Search Premier, EBSCOhost (accessed July 13, 2010).

35) Malgin AS and Ryazhskaya OG. 2008. "Neutrons from muons underground." Physics of Atomic Nuclei 71, no. 10 (October 2008): 1769-1781. Academic Search Premier, EBSCOhost (accessed July 13,2010$)$.

36) Matsumoto $\mathrm{H}$, et al. 2009. "The energy spectrum of primary cosmic rays estimated by using the arrival time spread of air shower particles." Nuclear Physics B Proceedings Supplement 196, (December 2009): 399-402. Academic Search Premier, EBSCOhost (accessed July 13, 2010).

37) *McNally F. "Simulating Light Production in IceTop." Carleton College (accessed July 17 2010). [Figure 3.]

38) Miyake S. 1963. "Empirical formula for range spectrum of cosmic ray $\mu$ mesons at sea level." J. Phys. Soc. Japan, 18, (1963) 1093

39) "Muon-assured defence." Economist 374, no. 8415 (February 26, 2005): 79-80. Academic Search Premier, EBSCOhost (accessed July 13, 2010).

40) *Percy JC and Rogers IW. 1977. "Cosmic ray intensities at shallow depths." Proc. 15th Int. Conf on Cosmic Rays, Volume 6. (1977) 99-103.

41) *Pevey, RE. “Lesson 2: Directions and Solid Angles." NE 406 Radiation Protection and Shielding. 1998. University of Tennessee. 27 Jul. 2010 $<$ http://web.utk.edu/ rpevey/NE406/lesson2.htm>.

42) Reyna D. 2008. "A Simple Parameterization of the Cosmic-Ray Muon Momentum Spectra at the Surface as a Function of Zenith Angle.” Elsevier. (eprint arXiv:hep-ph/0604145)

43) Ryazhskaya OG, Volkova LV, Zatespin GT, 2005. "Direct Cosmic Ray Muons and Atmospheric Neutrinos.” Nuclear Physics B (Proc. Suppl.) 143 (2005) 527.

44) Salazar H and Villaseñor L. 2006. "Astroparticle Physics: Detectors for Cosmic Rays." AIP Conference Proceedings 857, no. 2 (October 2006): 382-394. Academic Search Premier, EBSCOhost (accessed July 13, 2010).

45) Sdrolia A, Sfamba I, Liolios A, and Kitis G. 2010. "Cosmic Radiation Intensity Measurements Using TL Dosimeters at Various Mountain Altitudes." AIP Conference Proceedings 1203, no. 1 (January 21, 2010): 40-42. Academic Search Premier, EBSCOhost (accessed July 13, 2010).

46) *Thompson J. 1938. "Solar diurnal variation of cosmic-ray intensity as a function of latitude." Phys. Rev. 54, 93-96, 1938.

47) *Tiwari CM and Tiwari DP. 2008. "Characteristics of high energy cosmic ray diurnal anisotropy on day-to-day basis." Cosmic Research 46, no. 5 (September 2008): 465-468. Academic Search Premier, EBSCOhost (accessed July 14, 2010). 
48) Tsuji S, Katayama T, Okei K, Wada T, Yamamoto I, Yamashita Y. 1998. "Measurements of muons at sea level." J. Phys. G: Nucl. Part. Phys. 24 (1998) 1805.

49) Volkova LV and Saavedra O. 2009. "Prompt muon production in cosmic rays." Astroparticle Physics 32, no. 2 (September 2009): 136-139. Academic Search Premier, EBSCOhost (accessed July 13, 2010).

50) Voulgaris G, Kazanas S, and Chamilothoris I. 2010. "A Cosmic Ray Telescope For Educational Purposes." AIP Conference Proceedings 1203, no. 1 (January 21, 2010): 1433-1438. Academic Search Premier, EBSCOhost (accessed July 13, 2010)

51) Wang YF, Balic V, Gratta G, Fassò A, Roesler S, Ferrari A, 2001. "Predicting Neutron Production from Cosmic-ray Muons.” PACS numbers: 96.40.Tv, 14.60.Pq, 25.20.-x, 25.40.Sc

52) *Wolfram Research, Inc. 2008. “Mathematica Edition: Version 7.0.” Wolfram Research, Inc. Champaign Illinois. 2008.

53) Wolverton M. 2007. "Muons for Peace." Scientific American 297, no. 3 (September 2007): 2628. Academic Search Premier, EBSCOhost (accessed July 13, 2010).

54) Wright AG. 1973. "An accurate measurement of muon intensity at two depths underground." Proceedings of the $13^{\text {th }}$ International Conference on Cosmic Rays. Vol. 3, (1973): 1709-1714

55) *Wright AG. 1974. "A study of muons underground and their energy spectrum at sea level." J. Phys. A: Math., Nucl. Gen.. Vol. 7, No. 16, 1974. 


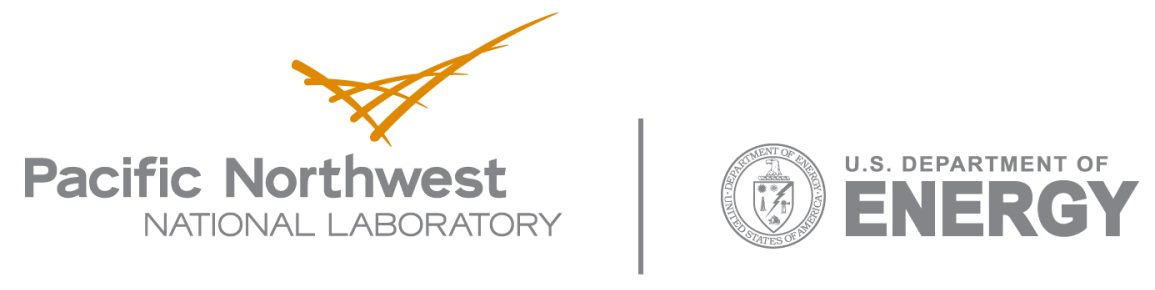

902 Battelle Boulevard

P.O. Box 999

Richland, WA 99352

1-888-375-PNNL (7665)

www.pnl.gov 